\title{
TYPE-Ia SUPERNOVA RATES TO REDSHIFT 2.4 FROM CLASH: THE CLUSTER LENSING AND SUPERNOVA SURVEY WITH HUBBLE
}

\author{
O. Graur ${ }^{1,2,3,4}$, S. A. Rodney ${ }^{1,20}$, D. Maoz ${ }^{2}$, A. G. Riess ${ }^{1,5}$, S. W. Jha ${ }^{6}$, M. Postman ${ }^{5}$, T. Dahlen ${ }^{5}$, T. W.-S. Holoien ${ }^{6}$, \\ C. McCully ${ }^{6}$, B. Patel ${ }^{6}$, L.-G. Strolger ${ }^{5}$, N. Benítez ${ }^{7}$, D. CoE $^{5}$, S. Jouvel ${ }^{8}$, E. Medezinski ${ }^{1}$, A. Molino ${ }^{7}$, \\ M. Nonino ${ }^{9}$, L. Bradley ${ }^{5}$, A. Koekemoer ${ }^{5}$, I. Balestra ${ }^{9,10}$, S. B. CenKo ${ }^{11,12}$, K. I. ClubB ${ }^{12}$, M. E. Dickinson ${ }^{13}$, \\ A. V. Filippenko ${ }^{12}$, T. F. Frederiksen ${ }^{14}$, P. GARnAvich ${ }^{15}$, J. HJorth ${ }^{14}$, D. O. Jones ${ }^{1}$, B. LeibundGut $^{16,17}$, T. Matheson $^{13}$, \\ B. Mobasher ${ }^{18}$, P. Rosati ${ }^{16}$, J. M. Silverman ${ }^{19,21}$, V. U ${ }^{18}$, K. JedruszczUK ${ }^{3}$, C. Li $^{3}$, K. Lin ${ }^{3}$, M. Mirmelstein ${ }^{2}$, \\ J. NeustadT ${ }^{3}$, A. Ovadia ${ }^{3}$, And E. H. Rogers ${ }^{3}$ \\ ${ }^{1}$ Department of Physics and Astronomy, The Johns Hopkins University, Baltimore, MD 21218, USA; orgraur@jhu.edu \\ ${ }^{2}$ School of Physics and Astronomy, Tel-Aviv University, Tel-Aviv 69978, Israel \\ ${ }^{3}$ Department of Astrophysics, American Museum of Natural History, New York, NY 10024, USA \\ ${ }^{4}$ CCPP, New York University, 4 Washington Place, New York, NY 10003, USA \\ ${ }^{5}$ Space Telescope Science Institute, Baltimore, MD 21218, USA \\ ${ }^{6}$ Department of Physics and Astronomy, Rutgers, The State University of New Jersey, Piscataway, NJ 08854, USA \\ ${ }^{7}$ Instituto de Astrofísica de Andalucía (CSIC), E-18080 Granada, Spain \\ ${ }^{8}$ Institut de Ciencies de l'Espai, (IEEC-CSIC), E-08193 Bellaterra (Barcelona), Spain \\ ${ }^{9}$ INAF-Osservatorio Astronomico di Trieste, I-34143 Trieste, Italy \\ ${ }^{10}$ INAF-Osservatorio Astronomico di Capodimonte, Via Moiariello 16, I-80131 Napoli, Italy \\ ${ }^{11}$ Astrophysics Science Division, NASA Goddard Space Flight Center, Mail Code 661, Greenbelt, MD 20771, USA \\ ${ }^{12}$ Department of Astronomy, University of California, Berkeley, CA 94720-3411, USA \\ ${ }^{13}$ National Optical Astronomy Observatory, 950 North Cherry Avenue, Tucson, AZ 85719, USA \\ ${ }^{14}$ Dark Cosmology Centre, Niels Bohr Institute, University of Copenhagen, Juliane Maries Vej 30, DK-2100 Copenhagen, Denmark \\ ${ }^{15}$ Physics Department, University of Notre Dame, Notre Dame, IN 46556, USA \\ ${ }^{16}$ European Southern Observatory, D-85748 Garching bei München, Germany \\ ${ }^{17}$ Excellence Cluster Universe, Technische Universitaet Muenchen, D-85748 Garching, Germany \\ ${ }^{18}$ Department of Physics and Astronomy, University of California, Riverside, CA 92521, USA \\ ${ }^{19}$ Department of Astronomy, University of Texas, Austin, TX 78712-0259, USA \\ Received 2013 October 10; accepted 2014 January 3; published 2014 February 10
}

\begin{abstract}
We present the supernova (SN) sample and Type-Ia SN (SN Ia) rates from the Cluster Lensing And Supernova survey with Hubble (CLASH). Using the Advanced Camera for Surveys and the Wide Field Camera 3 on the Hubble Space Telescope (HST), we have imaged 25 galaxy-cluster fields and parallel fields of non-cluster galaxies. We report a sample of $27 \mathrm{SNe}$ discovered in the parallel fields. Of these SNe, $\sim 13$ are classified as SN Ia candidates, including four SN Ia candidates at redshifts $z>1.2$. We measure volumetric SN Ia rates to redshift 1.8 and add the first upper limit on the SN Ia rate in the range $1.8<z<2.4$. The results are consistent with the rates measured by the HST/GOODS and Subaru Deep Field SN surveys. We model these results together with previous measurements at $z<1$ from the literature. The best-fitting SN Ia delay-time distribution (DTD; the distribution of times that elapse between a short burst of star formation and subsequent SN Ia explosions) is a power law with an index of

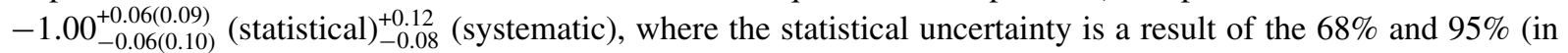
parentheses) statistical uncertainties reported for the various SN Ia rates (from this work and from the literature), and the systematic uncertainty reflects the range of possible cosmic star-formation histories. We also test DTD models produced by an assortment of published binary population synthesis (BPS) simulations. The shapes of all BPS double-degenerate DTDs are consistent with the volumetric SN Ia measurements, when the DTD models are scaled up by factors of 3-9. In contrast, all BPS single-degenerate DTDs are ruled out by the measurements at $>99 \%$ significance level.
\end{abstract}

Key words: supernovae: general - surveys - white dwarfs

Online-only material: color figures

\section{INTRODUCTION}

Although Type-Ia supernovae ( $\mathrm{SNe}$ Ia) have been used to measure extragalactic distances and thus reveal the accelerating expansion of the universe (Riess et al. 1998; Schmidt et al. 1998; Perlmutter et al. 1999), the nature of the stellar system that leads to these explosions remains unclear (see review by Howell 2011). The current consensus is that the progenitor is a

\footnotetext{
${ }^{20}$ Hubble Fellow.

${ }^{21}$ NSF Astronomy and Astrophysics Postdoctoral Fellow.
}

carbon-oxygen white dwarf (CO WD) that accretes matter from a binary companion until the pressure or temperature somewhere in the WD become high enough to ignite the carbon and lead to a thermonuclear explosion of the WD (Leibundgut 2000). Different scenarios have been proposed to explain the nature of the binary companion and the process of mass accretion. The leading scenarios are the single-degenerate (SD) scenario (Whelan \& Iben 1973), in which the binary companion is either a main-sequence star, a subgiant just leaving the main sequence, a red giant, or a stripped "He star," and the WD accretes mass from the secondary through Roche-lobe overflow or a stellar wind. 
In the double-degenerate (DD) scenario (Iben \& Tutukov 1984; Webbink 1984), the companion is a second CO WD and the two WDs merge due to loss of energy and angular momentum to gravitational waves.

Each of these scenarios predicts a different form of the distribution of times that elapse between a short burst of star formation and any subsequent SN Ia events, known as the delaytime distribution (DTD; see Wang \& Han 2012 and Hillebrandt et al. 2013 for recent reviews). The DTD can be thought of as a transfer function connecting the star-formation history (SFH) of a specific stellar environment and that environment's SN Ia rate. Thus, by measuring the $\mathrm{SN}$ Ia rate and comparing it to the SFH, one might reconstruct the DTD. The SN Ia DTD has been recovered using several techniques applied to different SN samples collected from different types of stellar environments (see review by Maoz \& Mannucci 2012). The emerging picture is that of a power-law DTD with an index of $\sim-1$, a form that arises naturally from the DD scenario, although combinations of DTDs from a DD channel and a SD channel cannot be ruled out. One method to recover the DTD, $\Psi(t)$, is to measure the SN Ia rate, $R_{\mathrm{Ia}}(t)$, as a function of cosmic time $t$ in field galaxies, and compare them to the cosmic SFH, $S(t)$ :

$$
R_{\mathrm{Ia}}(t)=\int_{0}^{t} S(t-\tau) \Psi(\tau) d \tau .
$$

Measurements of the volumetric SN Ia rates (i.e., the SN Ia rates per unit volume) in field galaxies agree out to $z \approx 1$. Graur et al. (2011, G11) provide a compilation of all SN Ia rates measured up to 2011, and later measurements are presented by Krughoff et al. (2011), Perrett et al. (2012), Barbary et al. (2012), Melinder et al. (2012), and Graur \& Maoz (2013). Volumetric SN Ia rate measurements were first extended to $z>1$ by Dahlen et al. (2004), with additional data analyzed by Dahlen et al. (2008, D08), using the Hubble Space Telescope (HST) to survey the GOODS fields (Giavalisco et al. 2004; Riess et al. 2004). The HST/GOODS survey discovered 20 SNe Ia at $1<z<1.4$ and 3 at $1.4<z<1.8$. G11 conducted a SN survey in the Subaru Deep Field (SDF) using the $8.2 \mathrm{~m}$ Subaru telescope and discovered $27 \mathrm{SN}$ Ia candidates at $1<z<1.5$ and 10 at $1.5<z<2$.

The SN Ia rate uncertainties at $z>1$, and especially at $z>1.5$, are dominated by small-number statistics. The three $z>1.4 H S T /$ GOODS SNe Ia were discovered in host galaxies having a spectroscopic redshift (spec- $z$ ) and no active galactic nucleus (AGN) activity. On the other hand, the classification of the larger SDF SN sample from G11 relies on photometric redshift (photo-z) measurements that might be systematically biased toward high redshifts (see their Section 4.2). While G11 also used several methods to weed out interloping AGNs, there could still be some AGN contamination because each $\mathrm{SN}$ in the SDF sample was only observed on one epoch. The HST/ GOODS sample, while smaller than the SDF sample, suffers from lower systematic uncertainties owing to the spectroscopic classification of the SN host galaxies and measurements of their redshifts, and a better sampling of the SN light curves. Of the 10 $z>1.5 \mathrm{SN}$ host galaxies in the SDF sample, only one galaxy has so far had its redshift and lack of AGN activity confirmed spectroscopically (Frederiksen et al. 2012).

Although the GOODS and SDF $z>1 \mathrm{SN}$ Ia rates are consistent, their interpretation differs between the two groups. Based on the GOODS data, Dahlen et al. (2004, 2008) argued that the $\mathrm{SN}$ Ia rate declined at $z>0.8$. Fitting this declining $\mathrm{SN}$ Ia rate evolution, Strolger et al. $(2004,2010)$ surmised that the
DTD is confined to delay times of 3-4 Gyr. In contrast, based on the SDF data, G11 found that the SN Ia rate evolution does not decline at high redshifts, but rather levels off, as would be expected of a power-law DTD.

Two new SN surveys are attempting to resolve this conflict. These surveys are components of two 3 yr HST Multi-Cycle Treasury programs that use the Advanced Camera for Surveys (ACS) and the new Wide Field Camera 3 (WFC3). Results from the Cosmic Assembly Near-infrared Deep Extragalactic Legacy Survey (CANDELS; Grogin et al. 2011; Koekemoer et al. 2011) will be reported by Rodney et al. (2014). Here, we describe results from the Cluster Lensing And Supernova survey with Hubble (CLASH; Postman et al. 2012). CLASH imaged 25 galaxy clusters in 16 broad-band filters from the nearultraviolet (NUV) to the near-infrared (NIR) with the ACS and WFC3 cameras working in parallel mode. While one camera was pointed at the galaxy cluster, the other one was used to observe a parallel field far enough from the galaxy cluster so as not to be significantly affected by strong lensing.

In this work, we report a sample of $27 \mathrm{SNe}$ discovered in the parallel fields of the 25 CLASH galaxy clusters. In Section 2, we describe the CLASH observations and our imaging and spectroscopic follow-up program. We report our SN sample in Section 3, where we also conduct detectionefficiency simulations and classify the SNe. Using our SN Ia sample, we measure $\mathrm{SN}$ Ia rates out to $z \approx 2.4$ in Section 4 and use them to test different forms of the DTD in Section 5. Finally, we summarize our results in Section 6. Throughout this work, we assume a $\Lambda$-cold-dark-matter cosmological model with parameters $\Omega_{\Lambda}=0.7, \Omega_{m}=0.3$, and $H_{0}=70 \mathrm{~km} \mathrm{~s}^{-1}$ $\mathrm{Mpc}^{-1}$. Unless noted otherwise, all magnitudes are on the Vega system.

We designate our SN candidates according to the cluster and year in which they were discovered and the first three letters of the nickname given to them for internal tracking purposes. For example, CLI11Had is a CLASH (CL) SN that was discovered in one of the parallel fields around the ninth (or Ith) cluster, MACS0717.5+3745, in 2011, and was nicknamed "Hadrian." For the sake of brevity, we will henceforth refer to our SN candidates simply as SNe.

\section{OBSERVATIONS}

\subsection{Imaging}

The CLASH observation strategy is described in detail by Postman et al. (2012). During Cycles 18-20, CLASH observed 25 galaxy clusters in the redshift range $0.187-0.890$. The central region of each galaxy cluster was imaged with 16 broad-band filters from the NUV to the NIR using the ACS and WFC3 cameras on HST. In ACS, we used the Wide Field Channel (WFC), with a field of view of $202^{\prime \prime} \times 202^{\prime \prime}$ and a pixel scale of 0 .'05 pixel $^{-1}$. WFC3 includes two detectors: an infrared channel (WFC3-IR) with a field of view of $123^{\prime \prime} \times 136^{\prime \prime}$ and a scale of $0^{\prime \prime} 13$ pixel $^{-1}$; and an ultraviolet-visible channel (WFC3-UVIS) with a field of view of $162^{\prime \prime} \times 162^{\prime \prime}$ and a scale of $0^{\prime \prime} .04$ pixel $^{-1}$.

The orientation of HST and the cadence between succeeding visits to the galaxy cluster ("prime") field were chosen so that two ACS and two WFC3 parallel fields would each be observed on four separate occasions, with a median cadence of 18 days. Each visit to a WFC3 parallel field consisted of one orbit comprising two F160W filter exposures and one exposure in filters F125W and F350LP each (filter+system central wavelengths $\lambda_{0} \approx 15,369,12,486$, and $5846 \AA$, respectively). Visits 
Table 1

Typical Exposure Times for CLASH Parallel Fields

\begin{tabular}{lcccc}
\hline \hline Camera & Filter & Exposures & $\begin{array}{c}\text { Total Time } \\
(\mathrm{s})\end{array}$ & $\begin{array}{c}\text { 5 } \sigma \text { Limiting Magnitude } \\
\text { (Vega mag) }\end{array}$ \\
\hline ACS-WFC & F850LP & 4 & 1500 & 25.0 \\
& & 6 & 3600 & 25.4 \\
ACS-WFC & F775W & 1 & 400 & 25.7 \\
& & 2 & 700 & 25.9 \\
WFC3-IR & F160W & 2 & 1200 & 25.4 \\
WFC3-IR & F125W & 1 & 700 & 25.7 \\
WFC3-UVIS & F350LP & 1 & 650 & 27.5 \\
\hline
\end{tabular}

to the ACS parallel fields consisted of one orbit when the prime field was imaged with either the ACS or WFC3-IR cameras and two orbits when the prime field was imaged with the WFC3UVIS camera. During single-orbit visits, the parallel ACS orbit comprised four F850LP filter exposures and one F775W filter exposure (filter+system central wavelengths $\lambda_{0} \approx 9445$ and $7764 \AA$, respectively). When the ACS parallel fields were imaged over two orbits, they consisted of six F850LP and two F775W exposures. These filters, the reddest in each camera, were chosen to detect high-redshift SNe. The F350LP band was added to the WFC3 observations for additional color information to aid in the classification of any $\mathrm{SNe}$ discovered in those fields. The HST angular resolution in our search bands is $\sim 0$ '. 10 and $\sim 00^{\prime \prime} 17$ in F850LP and F160W, respectively, slightly larger than the pixel scales of their respective cameras. Table 1 lists the typical exposure times and $5 \sigma$ limiting magnitudes reached in each of these filters.

The limiting magnitude in each filter was calculated using the method outlined in Kashikawa et al. (2004): we conducted aperture photometry on hundreds of blank regions in the image, fit a Gaussian to the negative side of the resultant histogram (as the positive tail could be contaminated by light from the sources in the image), and treated the standard deviation of the fit as an estimate of the average noise in the image. We used circular apertures with radii of 4,3 , and 5 pixels, which correspond to $0.20,0.27$, and $0^{\prime} .20$ in the pixel scale to which we drizzle the images taken with ACS, WFC3-IR, and WFC3-UVIS: 0.05, 0.09 , and 0.04 arcsec pixel ${ }^{-1}$, respectively.

An additional 52 HST orbits were allocated for followup imaging or slitless spectroscopy of targets of opportunity, such as high-redshift SNe Ia. This cache of orbits was added to the 150 similar HST orbits allocated to the CANDELS program, for a sum of 202 follow-up orbits for the combined CLASH+CANDELS SN survey (PI: A. Riess).

Our HST reduction and image-subtraction pipeline is described in detail by Rodney et al. (2014). Briefly, the raw HST images were first calibrated using the STSDAS $^{22}$ calibration tools. The calibrations include bias correction, dark subtraction, and flat fielding. In the case of WFC3-IR images, "up-the-ramp" fitting was used to remove cosmic ray (CR) events. Chargetransfer efficiency losses in the ACS images were corrected using the algorithm of Anderson \& Bedin (2010). Next, the subexposures in each filter were combined using MULTIDRIZZLE (Koekemoer et al. 2003). This stage also removed the geometrical distortion of the HST focal plane. For each filter, we created "template" images comprised of all previous observations in the same filter. This means that some SN light may be included in

$\overline{22}$ http://www.stsci.edu/institute/software_hardware/pyraf/stsdas the template images, which we take into account in Section 4. Finally, we subtracted the template images from the drizzled "target" images to produce the difference images that were then searched for SNe. Owing to the stable point-spread-function (PSF) of HST, we did not need to degrade the PSF of either the target or template images to match the PSF of the images, as done in ground-based SN surveys (e.g., G11).

Most of the CLASH galaxy clusters were observed in the $B, V$, $R_{c}, I_{c}$, and $z^{\prime}$ bands with Suprime-Cam (Miyazaki et al. 2002) at the prime focus of the $8.2 \mathrm{~m}$ Subaru telescope, for the purpose of measuring the amount of shear induced on background galaxies by weak lensing from the galaxy cluster, and for deriving the photometric redshifts of the galaxies in the CLASH parallel fields. For an example of such observations of the CLASH galaxy cluster MACS1206, and a description of their reduction, see Umetsu et al. (2012).

\subsection{Spectroscopy}

The host galaxies of all SN candidates, and in several cases the SNe themselves, were followed up with spectroscopic observations from several ground-based observatories, as detailed below, or with HST slitless spectroscopy, using the G800L ACS grism spectrograph. The ground-based observatories and instruments used for this work were the Low Resolution Imaging Spectrometer (LRIS; Oke et al. 1995) and the DEep Imaging Multi-Object Spectrograph (DEIMOS; Faber et al. 2003) on the Keck I and II $10 \mathrm{~m}$ telescopes, respectively; the Gemini Multi-Object Spectrograph (GMOS; Hook et al. 2003) on the Gemini North and South telescopes (GeminiN and GeminiS, respectively); the Multi-Object Double Spectrograph (MODS; Pogge et al. 2010) on the Large Binocular Telescope (LBT); and the FOcal Reducer and low dispersion Spectrograph (FORS; Appenzeller et al. 1998), the VIsible MultiObject Spectrograph (VIMOS; Le Fèvre et al. 2003), and the X-shooter spectrograph (Vernet et al. 2011) on the Very Large Telescope (VLT). Table 2 details which instruments were used to obtain spectra of each SN host galaxy. Several examples of SN host-galaxy spectra are shown in Figure 1. The spectra of the SNe CLF11Ves, CLI11Had, and CLY13Pup are shown in Figure 2.

\section{SUPERNOVA SAMPLE}

In our survey, $\mathrm{SNe}$ can be discovered if they either brighten or decline between one search epoch and the next. By a "brightening" SN we do not mean that the $\mathrm{SN}$ is necessarily caught on the rising part of its light curve, but rather any case in which the discovery flux is higher than the template flux. As a result of the cadence of our survey, it is easier to discover $\mathrm{SNe}$ either on the rise or near peak, as in these cases the template image will contain no SN flux. In contrast, SNe caught while on the decline will invariably have some flux in all our images, thus reducing the flux in the difference image and consequently their probability of detection. We have discovered a total of 20 brightening and 7 declining SNe in the parallel fields of the 25 CLASH clusters. Of these, 18 were discovered in the ACS and 9 in the WFC3 fields. Nineteen (or 70\%) of the SN host galaxies have spectroscopic redshifts, as detailed below in Section 3.3. We classify half of this sample as SNe Ia, four of which are at $z>1.2$. Our SN sample is summarized in Table 2 and displayed in Figure 3.

We have discovered 12 additional SN candidates in the prime fields. However, as the effects of gravitational lensing must be taken into account to properly classify any $\mathrm{SNe}$ discovered 
Table 2

SNe Discovered in the Parallel Fields of the 25 CLASH Galaxy Clusters

\begin{tabular}{|c|c|c|c|c|c|c|c|c|}
\hline $\begin{array}{l}\text { ID } \\
\text { (1) }\end{array}$ & $\begin{array}{l}\text { Nickname } \\
\text { (2) }\end{array}$ & $\begin{array}{c}\alpha(\mathrm{h} \mathrm{m} \mathrm{s}) \\
\text { (3) }\end{array}$ & $\begin{array}{c}\delta\left({ }^{\circ \prime \prime \prime}\right) \\
(4)\end{array}$ & $\begin{array}{l}P(\mathrm{Ia})_{w p} \\
\quad(5)\end{array}$ & $\begin{array}{l}P(\mathrm{Ia})_{n p} \\
\quad(6)\end{array}$ & $\begin{array}{l}\text { Photo- } z \\
\text { (7) }\end{array}$ & $\begin{array}{c}\text { Spec- } z \\
(8)\end{array}$ & $\begin{array}{c}\text { Spec- } z \text { Source } \\
\text { (9) }\end{array}$ \\
\hline CLA10Cal & Caligula & $02: 48: 25.740$ & $-03: 33: 08.37$ & $0.95_{-0.14}^{+0.03}$ & $0.98_{-0.00}^{+0.00}$ & $1.68_{-0.15}^{+0.15}$ & $\cdots$ & (VLT+X-shooter) \\
\hline CLA10Ner & Nero & $02: 47: 40.180$ & $-03: 32: 53.29$ & $0.76_{-0.26}^{+0.09}$ & $0.82_{-0.08}^{+0.01}$ & $0.32_{-0.01}^{+0.08}$ & 0.362 & Keck+DEIMOS \\
\hline CLB11Oth & Otho & $11: 49: 56.745$ & $+22: 18: 42.87$ & $0.79_{-0.14}^{+0.06}$ & $0.89_{-0.01}^{+0.00}$ & $0.86_{-0.20}^{+0.15}$ & 0.962 & VLT+FORS2 \\
\hline CLC11Tit & Titus & $17: 22: 52.985$ & $+32: 07: 25.74$ & $0.89_{-0.08}^{+0.03}$ & $0.94_{-0.01}^{+0.01}$ & $0.70_{-0.05}^{+0.05}$ & 0.839 & Keck+LRIS \\
\hline CLD11Cla & Claudius & $12: 06: 08.868$ & $-08: 42: 54.73$ & $0.13_{-0.13}^{+0.24}$ & $0.19_{-0.19}^{+0.18}$ & $0.24_{-0.04}^{+0.07}$ & . & $(\mathrm{VLT}+\mathrm{FORS} 2 ; \mathrm{Keck}+\mathrm{LRIS} ; \mathrm{ACS}+\mathrm{G} 800 \mathrm{~L})$ \\
\hline CLE11Aug & Augustus & $13: 47: 12.802$ & $-11: 42: 28.97$ & $0.03_{-0.03}^{+0.06}$ & $0.06_{-0.06}^{+0.05}$ & $0.34_{-0.03}^{+0.10}$ & 0.329 & GeminiS+GMOS \\
\hline CLF11Ves & Vespasian & $21: 29: 42.612$ & $-07: 41: 48.08$ & $0.97_{-0.04}^{+0.01}$ & $0.98_{-0.01}^{+0.00}$ & $1.31_{-0.10}^{+0.05}$ & 1.22 & ACS+G800L; Keck+DEIMOS \\
\hline CLF11Dom & Domitian & $21: 29: 53.224$ & $-07: 40: 56.95$ & $0.63_{-0.40}^{+0.16}$ & $0.71_{-0.24}^{+0.06}$ & $0.71_{-0.09}^{+0.14}$ & $\ldots$ & $\ldots$ \\
\hline CLH11Tra & Trajan & $21: 39: 46.036$ & $-23: 38: 34.71$ & $1.00_{-0.00}^{+0.00}$ & $1.00_{-0.00}^{+0.00}$ & $1.41_{-0.11}^{+0.14}$ & $\ldots$ & (VLT+VIMOS; Keck+DEIMOS) \\
\hline CLI11Had & Hadrian & $07: 17: 20.115$ & $+37: 49: 54.54$ & $1.00_{-0.00}^{+0.00}$ & $1.00_{-0.00}^{+0.00}$ & $0.21_{-0.03}^{+0.03}$ & 0.261 & Keck+LRIS \\
\hline CLK11Bur & Burgundy & $06: 49: 13.878$ & $+70: 13: 17.91$ & $0.00_{-0.00}^{+0.00}$ & $0.00_{-0.00}^{+0.00}$ & $0.27_{-0.05}^{+0.05}$ & 0.281 & Keck+LRIS \\
\hline CLI11Piu & Antoninus Pius & $07: 17: 59.057$ & $+37: 40: 51.13$ & $0.00_{-0.00}^{+0.00}$ & $0.00_{-0.00}^{+0.00}$ & $0.18_{-0.02}^{+0.10}$ & 0.191 & GeminiS+GMOS; LBT+MODS \\
\hline CLL12Aur & Marcus Aurelius & $11: 16: 07.758$ & $+01: 23: 44.60$ & $0.00_{-0.00}^{+0.00}$ & $0.00_{-0.00}^{+0.00}$ & $0.29_{-0.04}^{+0.10}$ & 0.271 & Keck+DEIMOS \\
\hline CLL12Luc & Lucius Verus & $11: 15: 57.141$ & $+01: 23: 19.62$ & $0.00_{-0.00}^{+0.00}$ & $0.00_{-0.00}^{+0.00}$ & $0.33_{-0.03}^{+0.06}$ & 0.36 & Keck+DEIMOS \\
\hline CLM12Com & Commodus & $08: 00: 52.385$ & $+36: 07: 37.31$ & $0.00_{-0.00}^{+0.00}$ & $0.00_{-0.00}^{+0.00}$ & $0.19_{-0.07}^{+0.05}$ & 0.207 & Keck+DEIMOS \\
\hline CLM12Car & Cardinal & $08: 00: 42.802$ & $+36: 07: 13.81$ & $0.22_{-0.22}^{+0.27}$ & $0.34_{-0.34}^{+0.16}$ & $0.47_{-0.05}^{+0.08}$ & 0.518 & Keck+DEIMOS \\
\hline CLP12Get & Geta & $21: 29: 23.918$ & $+00: 08: 24.77$ & $1.00_{-0.00}^{+0.00}$ & $1.00_{-0.00}^{+0.00}$ & $1.64_{-0.04}^{+0.04}$ & 1.64 & VLT+X-shooter; (VLT+VIMOS) \\
\hline CLR12Arm & Neill Armstrong & $01: 31: 30.231$ & $-13: 34: 39.13$ & $0.01_{-0.01}^{+0.02}$ & $0.01_{-0.01}^{+0.01}$ & $1.12_{-0.36}^{+0.63}$ & . & (VLT+VIMOS; Keck+LRIS) \\
\hline CLS12Mac & Macrinus & $04: 15: 47.671$ & $-24: 00: 23.77$ & $0.09_{-0.09}^{+0.16}$ & $0.23_{-0.22}^{+0.13}$ & $0.95_{-0.03}^{+0.08}$ & 1.034 & Keck+DEIMOS \\
\hline CLT12Ela & Elagabalus & $22: 48: 09.132$ & $-44: 35: 16.63$ & $0.61_{-0.10}^{+0.06}$ & $0.70_{-0.06}^{+0.07}$ & $0.53_{-0.04}^{+0.03}$ & 0.6058 & Keck+LRIS \\
\hline CLT12Ale & Alexander Severus & $22: 49: 20.961$ & $-44: 32: 47.94$ & $0.12_{-0.11}^{+0.18}$ & $0.13_{-0.10}^{+0.10}$ & $1.0_{-0.5}^{+2.0}$ & $\ldots$ & (VLT+FORS2) \\
\hline CLC12Thr & Thrax & $17: 22: 44.529$ & $+32: 03: 35.96$ & $0.00_{-0.00}^{+0.00}$ & $0.00_{-0.00}^{+0.00}$ & $0.23_{-0.04}^{+0.03}$ & $\ldots$ & $\ldots$ \\
\hline CLV12Gor & Gordian & $11: 57: 10.258$ & $+33: 42: 19.60$ & $0.00_{-0.00}^{+0.00}$ & $0.00_{-0.00}^{+0.00}$ & $0.37_{-0.14}^{+0.18}$ & 0.516 & Keck+DEIMOS \\
\hline CLY13Lil & Lilla & $13: 11: 03.822$ & $-03: 15: 50.66$ & $1.00_{-0.00}^{+0.00}$ & $1.00_{-0.00}^{+0.00}$ & $0.675_{-0.006}^{+0.001}$ & 0.661 & GeminiN+GMOS \\
\hline CLY13Pup & Pupienus & $13: 10: 43.130$ & $-03: 06: 13.08$ & $1.00_{-0.00}^{+0.00}$ & $1.00_{-0.00}^{+0.00}$ & $0.76_{-0.04}^{+0.04}$ & 0.804 & VLT+FORS2 \\
\hline CLY13Hos & Hostilian & $13: 10: 46.685$ & $-03: 05: 22.98$ & $0.92_{-0.08}^{+0.00}$ & $0.95_{-0.03}^{+0.00}$ & $0.90_{-0.08}^{+0.05}$ & 0.876 & Keck+DEIMOS; VLT+FORS2 \\
\hline CLY13Gal & Trebonianus Gallus & $13: 11: 02.495$ & $-03: 16: 54.91$ & $0.00_{-0.00}^{+0.00}$ & $0.00_{-0.00}^{+0.00}$ & $0.8-1.8^{\mathrm{a}}$ & & $(\mathrm{VLT}+\mathrm{FORS} 2)$ \\
\hline
\end{tabular}

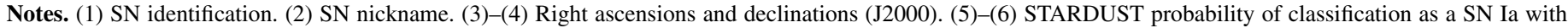

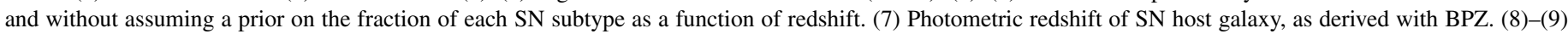
Spectroscopic redshift of SN host galaxy, where available, and its source. Parentheses indicate unsuccessful attempts or as yet unreduced data.

a The $68 \%$ confidence region derived from the photo- $z$ PDF of CLY13Gal, which has two prominent peaks at $z \approx 0.8$ and 1.6 .

behind the galaxy clusters, we leave their treatment to a future paper. The complete photometry of all $39 \mathrm{SNe}$ in our sample will appear in a future paper by O. Graur et al. (2014, in preparation).

\subsection{Candidate Selection}

The F850LP- and F160W-band subtraction images were simultaneously searched by eye and scanned with the sourceidentifying software SEXTRACTOR (Bertin \& Arnouts 1996) to identify variable objects. We set SEXTRACTOR to locate all objects that had at least four connected pixels with flux $3 \sigma$ above the local background level in both the regular subtraction image and in its negative, the latter in order to search for declining SNe. To increase the detection efficiency in the F160W band, the F160W- and F125W-band subtraction images were searched by eye simultaneously (by toggling between them), as some $\mathrm{SNe}$ may appear brighter in the F125W band (see, for example, the light-curve fit of CLI11Had in Figure 6). The F850LP- and F775W-band subtraction images were not similarly toggled due to the high CR contamination in the F775W-band subtraction image.

To be regarded as SN candidates, the variable objects had to pass the following criteria.
1. All objects with suspect residual shapes, such as the subtraction residuals of bright galaxy cores or objects with non-PSF shapes, were rejected.

2. The F850LP- and F160W-band images were comprised of several subexposures (four or six in the F850LP band and two in the F160W band). We used these subexposures to create separate subtraction images, which were then compared to the main subtraction image. The object had to appear in all of the subtraction images to be considered a likely candidate.

3. To be considered a declining SN, the object had to have a negative flux in the subtraction image and appear in both the target and template images. Objects that only appeared in the template images were discarded as either CRs or noise spikes.

4. Objects with suspect residual shapes in the F775W- or F125W-band subtraction images were flagged for inspection in the next search epoch. As a result of the cadence of the survey, any SNe detected in one of the first two search epochs would be visible in the other search epochs as well, so if the object under consideration did not reappear in a later epoch, it was rejected. No candidates were rejected if they did not appear in the F775W- or F125W-band images. 


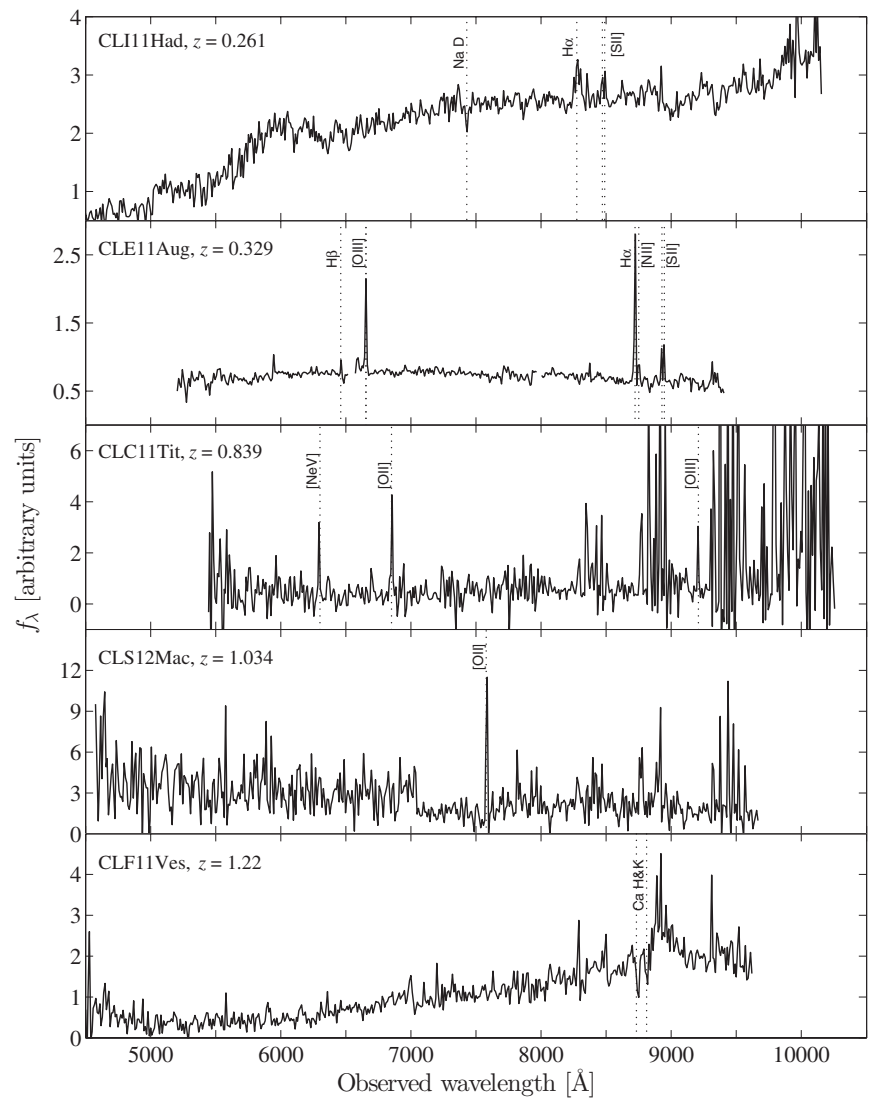

Figure 1. Examples of SN host-galaxy spectra. From top to bottom, we present spectra of the SN host galaxies CLI1 1Had, at $z=0.261$, taken with Keck+LRIS; CLE11Aug, at $z=0.329$, taken with GeminiS+GMOS (the gaps in this spectrum are the result of physical gaps between GMOS chips); CLC11Tit, at $z=0.839$, obtained with Keck+LRIS; CLS12Mac, at $z=1.034$, obtained with Keck+DEIMOS; and CLF11Ves, at $z=1.22$, obtained with Keck+DEIMOS. All spectra have been binned into $10 \AA$ wide bins. All flux units and have been arbitrarily scaled.

\subsection{Detection-efficiency Simulations}

In our survey, $\mathrm{SNe}$ can be missed because of many factors. Generally, the fainter the SN, the less likely it is to be detected above the background. On average, F775W-band images suffer from a background (composed of zodiacal light, earthshine, and airglow) level twice as high as F850LP-band images (Sokol et al. 2012). The main sources of background for WFC3-IR observations are earthshine and zodiacal light, with the latter being the dominant source. Both sources contribute less background at longer wavelengths (Giavalisco et al. 2002), but as our F160W exposures are roughly twice as long as the F125W exposures, they both display roughly the same number of background counts (Dressel 2012). There are other factors that affect the discovery probability of a $\mathrm{SN}$, such as its proximity to the core of its host galaxy ( $\mathrm{SNe}$ that explode close to the cores of their host galaxies are harder to discover due to the noise from the higher background and the residuals from imperfect image subtractions).

To test the effect of these and other factors on our detection efficiency, we planted $\sim 1000$ fake point sources in the raw images at the start of our reduction pipeline. The fake $\mathrm{SNe}$ were planted in random locations around galaxies chosen from SEXTRACTOR catalogs of the images following a Gaussian distribution centered on the center of the galaxy, as measured by SEXTRACTOR, with a standard deviation of $\sigma=2 R_{50}$, where

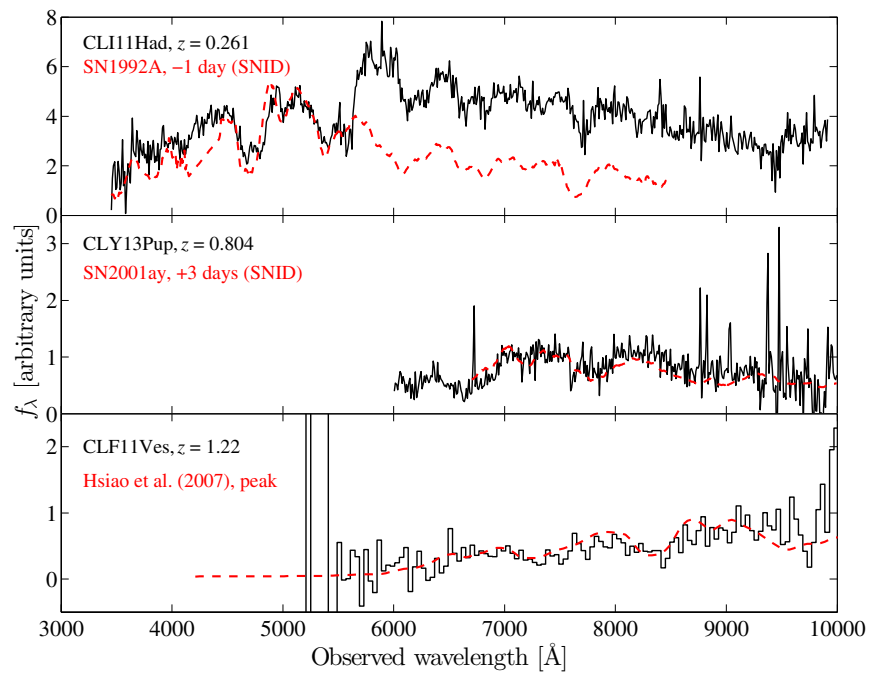

Figure 2. SN spectra. In black solid curves, we show the spectra of the $\mathrm{SNe}$ CLI11Had (top), at $z=0.261$, taken with Keck+LRIS; CLY13Pup (center), at $z=0.804$, taken with VLT+FORS2; and CLF11Ves (bottom), at $z=1.22$, taken with the HST G800L ACS grism spectrograph. Overlaid on the spectra as red dashed curves are examples of SNe Ia from the literature shifted to the same redshift and scaled so as to fit the data. The SN designation, along with that of the example SN Ia, are noted on the top left of each panel. The top and center spectra have been binned into $10 \AA$ wide bins, while the bottom spectrum has been binned into $80 \AA$ wide bins. All flux units are arbitrary. Beyond $5500 \AA$, the spectrum of CLI11 Had may be dominated by host-galaxy light, as no correction for host-galaxy light was performed during reductions.

(A color version of this figure is available in the online journal.)

$R_{50}$ is the radius that contains $50 \%$ of the galaxy light. This distribution assured that the fake $\mathrm{SNe}$ approximately followed the light of the galaxy and that a large number were planted in galaxy cores (e.g., Förster \& Schawinski 2008). Near the center of a bright galaxy, a SN could be obscured due to the increased Poisson noise and residual subtraction artifacts from small inter-epoch registration errors. This is evaluated in Rodney et al. (2014), where we find this effect to be negligible, with less than $2 \%$ of galaxies above $z=0.2$ exhibiting core residuals that could obscure a SN. The magnitudes of the fake SNe were drawn from flat distributions in F850LP and F160W in the range 22-28 mag. To simulate the appearance of real $\mathrm{SNe} \mathrm{Ia}$, the F775W and F125W magnitudes, respectively, were randomly chosen from a SN Ia simulation done with the SuperNova ANAlysis (SNANA ${ }^{23}$; Kessler et al. 2009b) software package, which was constructed to reflect a realistic spread of SN Ia colors in the redshift range $z=0-3$, with host-galaxy extinction according to values chosen from an exponential of the form $P\left(A_{V}\right)=e^{\left(-A_{V} / \tau_{V}\right)}$, with $\tau_{V}=0.7$, chosen to approximate the host-galaxy extinction model of Riello \& Patat (2005). This was done to ensure that the fake $\mathrm{SNe}$ resembled the colors of their real counterparts as close as possible, and was of importance mainly in the WFC3 fields, where the SN searchers toggled between the F160W and F125W difference images. The PSF was simulated using TINY TIM ${ }^{24}$ (Krist et al. 2011).

Figure 4 shows our detection efficiency, as a function of the brightness of the fake SNe, in the F850LP and F160W bands. The uncertainties of the measurements represent the $68 \%$ binomial confidence intervals. We follow Sharon et al. (2010)

\footnotetext{
23 http://sdssdp62.fnal.gov/sdsssn/SNANA-PUBLIC/

$24 \mathrm{http} / / /$ www.stsci.edu/hst/observatory/focus/TinyTim
} 

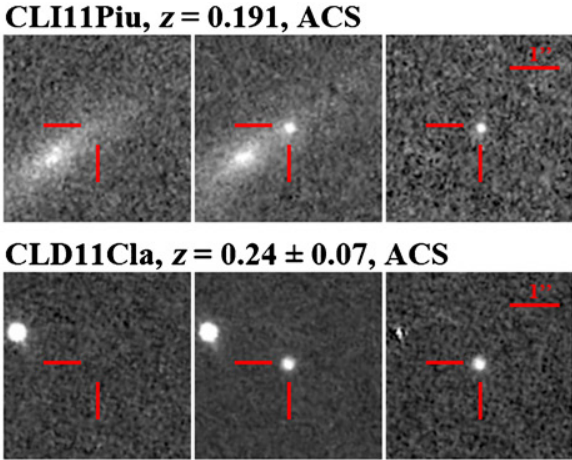

CLK11Bur, $z=0.281, A C S$

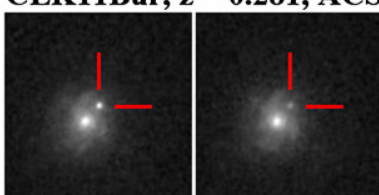

CLA10Ner, $z=0.362$, ACS

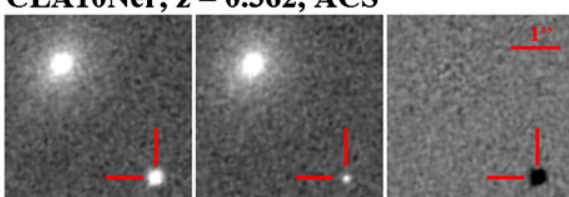

CLF11Dom, $z \sim 0.6, A C S$

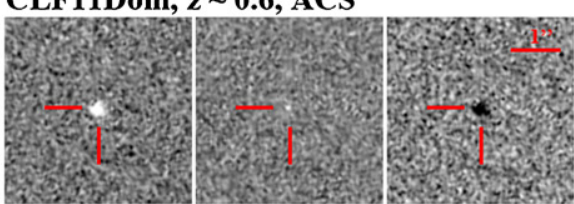

CLY13Gal, $z \sim 0.8$, WFC3

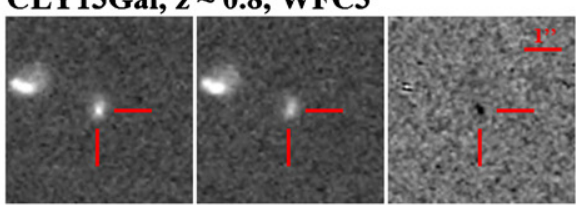

CLY13Hos, $z=0.876, A C S$

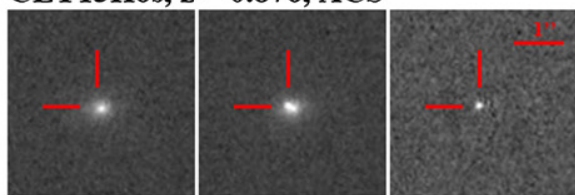

CLR12Arm, $z \sim 1$, WFC3

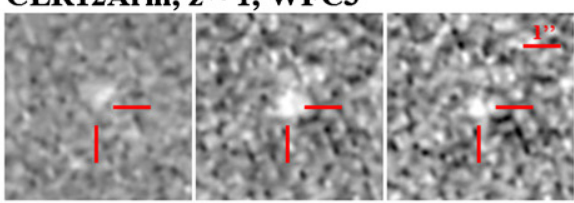

CLH11Tra, $z=1.41 \pm 0.14$, ACS

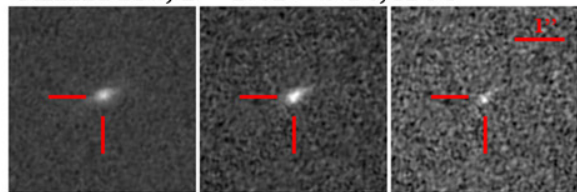

CLM12Com, $z=0.207, A C S$

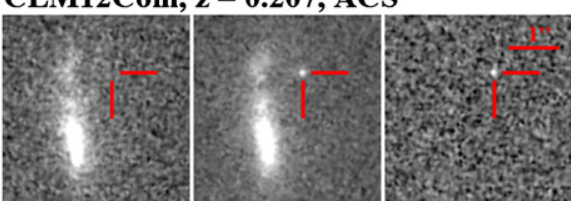

CLI11Had, $z=0.261$, WFC3

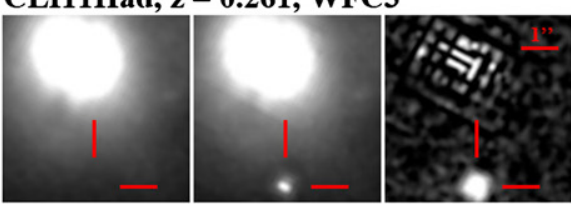

CLE11Aug, $z=0.329, \mathrm{ACS}$

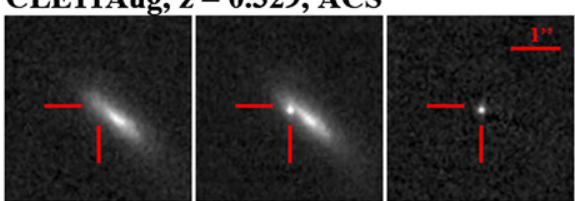

CLV12Gor, $z=0.516$, WFC3

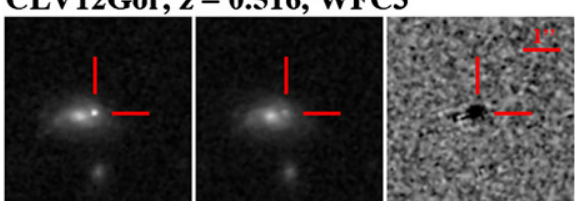

CLT12Ela, $z=0.6058, A C S$

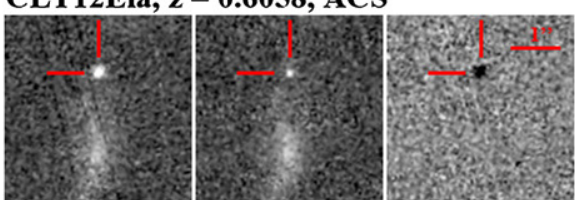

CLY13Pup, $z=0.804$, ACS

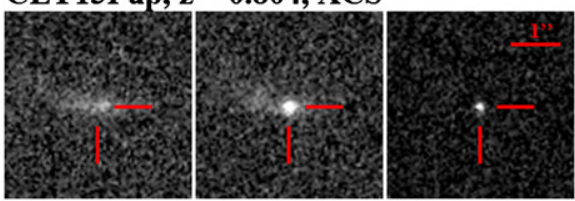

CLB10Oth, $z=0.962$, ACS

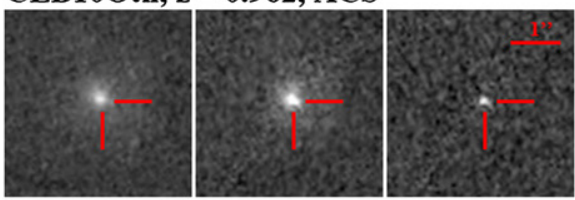

CLS12Mac, $z=1.034$, WFC3

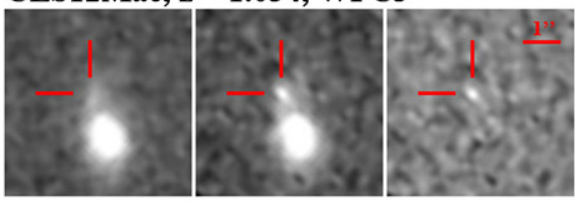

CLP12Get, $z=1.64 \pm 0.04$, WFC3

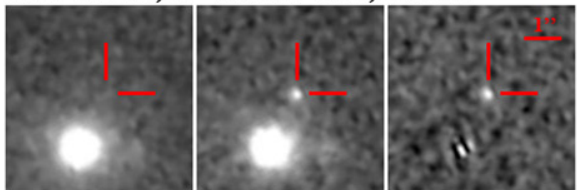

CLC12Thr, $z=0.23 \pm 0.04, A C S$

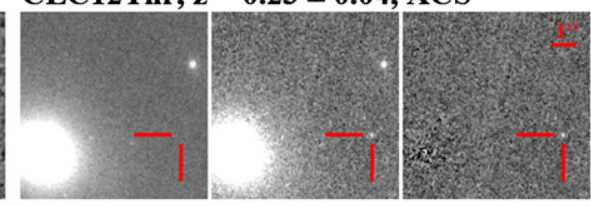

CLL12Aur, $z=0.271$, ACS

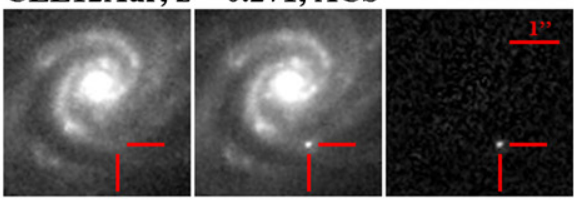

CLL12Luc, $z=0.36 \pm 0.06, A C S$

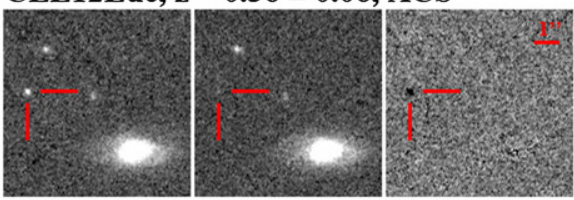

CLM12Car, $z=0.518, A C S$

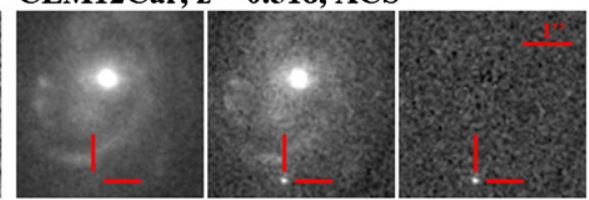

CLY13Lil, $z=0.661$, WFC3

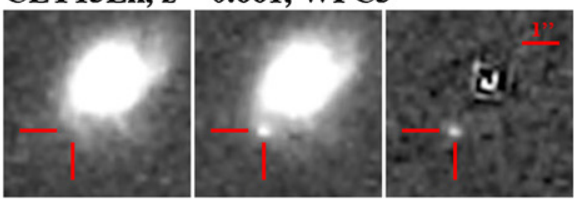

CLC11Tit, $z=0.839$, ACS

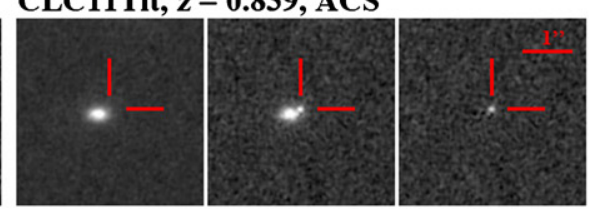

CLT12Ale, $z \sim 1$, WFC3

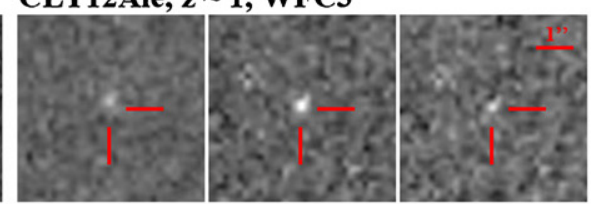

CLF11Ves, $z=1.22$, ACS

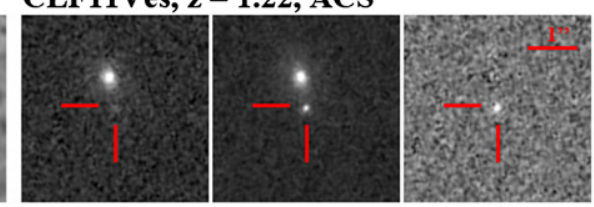

CLA10Cal, $z=1.68 \pm 0.15$, WFC3

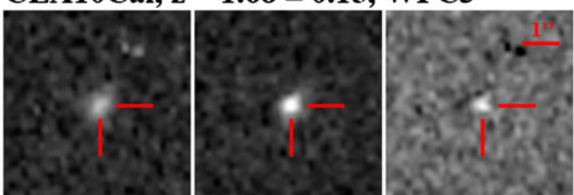

Figure 3. SNe discovered in the parallel fields of the CLASH clusters. North is up and east is left. In the triplet of tiles for each event, the left-hand tiles show the SN host galaxies without any SN light, whereas the center tiles display the SN host galaxy as imaged when the SN was first discovered. For the declining SNe CLK11Bur, CLL12Luc, CLA10Ner, CLV12Gor, CLF11Dom, CLT12Ela, and CLY13Gal, the left-hand and center tiles show the SN and host galaxy on the first and last visits to the field, respectively. The right-hand tiles show the subtraction in the F850LP or F160W bands for SNe discovered in the ACS or WFC3 parallel fields, respectively. The stretch of the images and the location of the SN differ from panel to panel in order to highlight host-galaxy properties. The header of each panel gives the designation of the SN along with its redshift and camera. Spectroscopic redshifts (cases with no uncertainties in $z$ noted) are given to three significant digits. Photometric redshifts are shown with their uncertainty; in cases where the photometric redshift is not well constrained, we note the approximate peak of the probability density function. (A color version of this figure is available in the online journal.) 


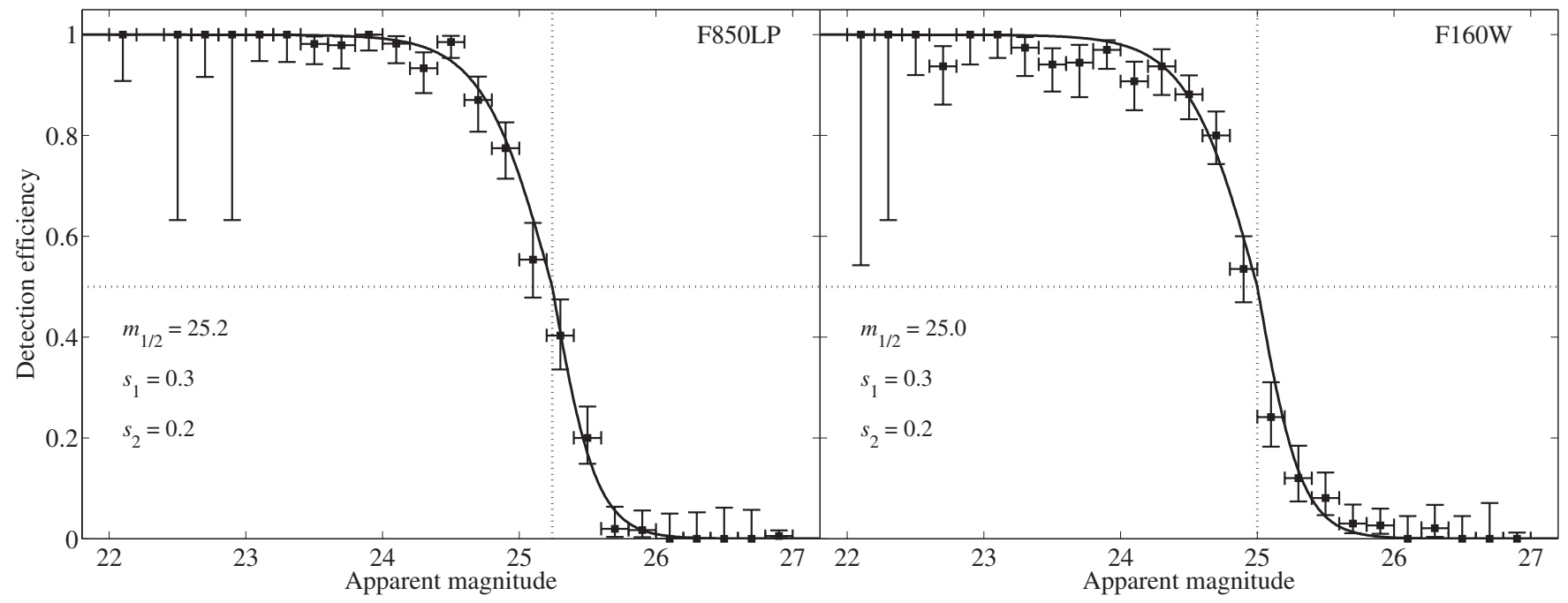

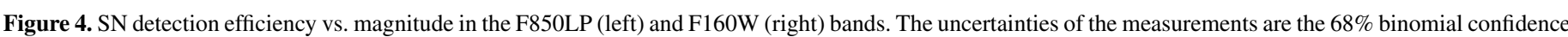
intervals. The dotted lines mark where the best-fit efficiency curves drop to 50\%, at 25.2 and 25.0 mag in the F850LP and F160W bands, respectively.

and fit the efficiency measurements with the function

$$
\eta\left(m ; m_{1 / 2}, s_{1}, s_{2}\right)= \begin{cases}\left(1+e^{\frac{m-m_{1 / 2}}{s_{1}}}\right)^{-1}, & m \leqslant m_{1 / 2} \\ \left(1+e^{\frac{m-m_{1 / 2}}{s_{2}}}\right)^{-1}, & m>m_{1 / 2},\end{cases}
$$

where $m$ is the magnitude in the F850LP band; $m_{1 / 2}$ is the magnitude at which the efficiency drops to 50\%; and $s_{1}$ and $s_{2}$ determine the range over which the efficiency drops from $100 \%$ to $50 \%$, and from $50 \%$ to 0 , respectively. Our detection efficiency drops to $50 \%$ at 25.2 and $25.0 \mathrm{mag}$ in the F850LP and F160W bands, respectively.

\subsection{Host-galaxy Redshifts}

Our classification method, as with most SN classification techniques, relies on a good knowledge of the redshift of either the SN or its host galaxy. As part of our survey strategy, we have endeavored to obtain spec- $z$ measurements of the host galaxies of all the SNe in our sample, mostly with ground-based observatories, as described above. Some of the SN host galaxies suspected of being at $z>1.2$ (CLD11Cla and CLF11Ves) were also followed up with HST slitless spectroscopy using the ACS G800L grism. At this time, we have acquired and reduced the spectra of 19 of the 27 SN host galaxies in our sample.

For the remaining eight $\mathrm{SN}$ host galaxies, we rely on photo- $z$ measurements. A complete description of our photo- $z$ technique appears in Jouvel et al. (2013) and A. Molino et al. (2014, in preparation). Here, we give only a brief description of this technique. The spec- $z$ and photo- $z$ values of the $\mathrm{SNe}$ in our sample are listed in Table 2 and shown in Figure 5.

We estimated the redshift and spectral type of all SN host galaxies with photometry obtained from deep Subaru images (in the $B, V, R_{c}, I_{c}$, and $z^{\prime}$ bands) and the Bayesian Photometric Redshift code (BPZ; Benítez 2000). For the host galaxies of $\mathrm{SNe}$ that were discovered in the WFC3 parallel fields, we also added galaxy photometry in the F125W and F160W bands. Some host galaxies were previously imaged by the Sloan Digital Sky Survey (SDSS; York et al. 2000), allowing us to include photometry in the $u^{\prime}, g^{\prime}, r^{\prime}, i^{\prime}$, and $z^{\prime}$ bands as well.

For each galaxy, BPZ calculates a likelihood, $L(z, T)$, as a function of redshift, $z$, and spectral type, $T$, comparing the observed colors of the galaxies with the template library, and then multiplies it by an empirical prior, $p(z, T \mid m)$, which depends on the galaxy magnitude in some reference band, $m$, yielding a full probability, $p(z, T)$, for each galaxy. The new version of BPZ (N. Benítez 2014, in preparation) includes a new template library comprising six spectral energy distribution templates originally from PEGASE (Fioc \& Rocca-Volmerange 1997) and four early-type templates from Polletta et al. (2007). The PEGASE templates were recalibrated using the Wuyts et al. (2008) FIREWORKS photometry and spectroscopic redshifts to optimize its performance together with the new early-type galaxy templates. In total, we use five templates for early-type galaxies, two for intermediate galaxies, and four for starburst galaxies. The prior was calibrated using the GOODS-MUSIC (Grazian et al. 2006), Ultra Deep Field (UDF) (Coe et al. 2006), and COSMOS (Ilbert et al. 2009) samples. Despite its compactness and simplicity, this library produces results which are comparable or slightly better than the best available photo- $z$ methods (see the method comparison in Hildebrandt et al. 2010), which often include template libraries that are many times larger. As a result of the high-quality HST imaging used for its calibration and using an approach similar to that developed by Coe et al. (2006), the representation of typical galaxy colors provided by this library can be used to calibrate ground-based photometry to an accuracy of $\sim 2 \%$ (A. Molino et al. 2014, in preparation).

\subsection{Supernova Classification}

We classify our SNe into SNe Ia, SNe Ib/c, or SNe II by fitting light curves to their multi-band photometry using a Bayesian approach first introduced by Jones et al. (2013), where it was used to classify the CANDELS SN UDS10Wil. The full description of this classification technique, named the Supernova Taxonomy And Redshift Determination Using SNANA Templates (STARDUST), along with a detailed examination of any systematic biases it might introduce, will appear in a future paper by S. A. Rodney et al. (2014, in preparation). Briefly, for each SN we calculate the probability that it is a $\mathrm{SN}$ Ia, $P(\mathrm{Ia})$, by comparing the observed fluxes (in all available bands and epochs) to lightcurve models generated using the SNANA simulation package. We classify a SN as a SN Ia if $P(\mathrm{Ia}) \geqslant 0.5$. However, as detailed 


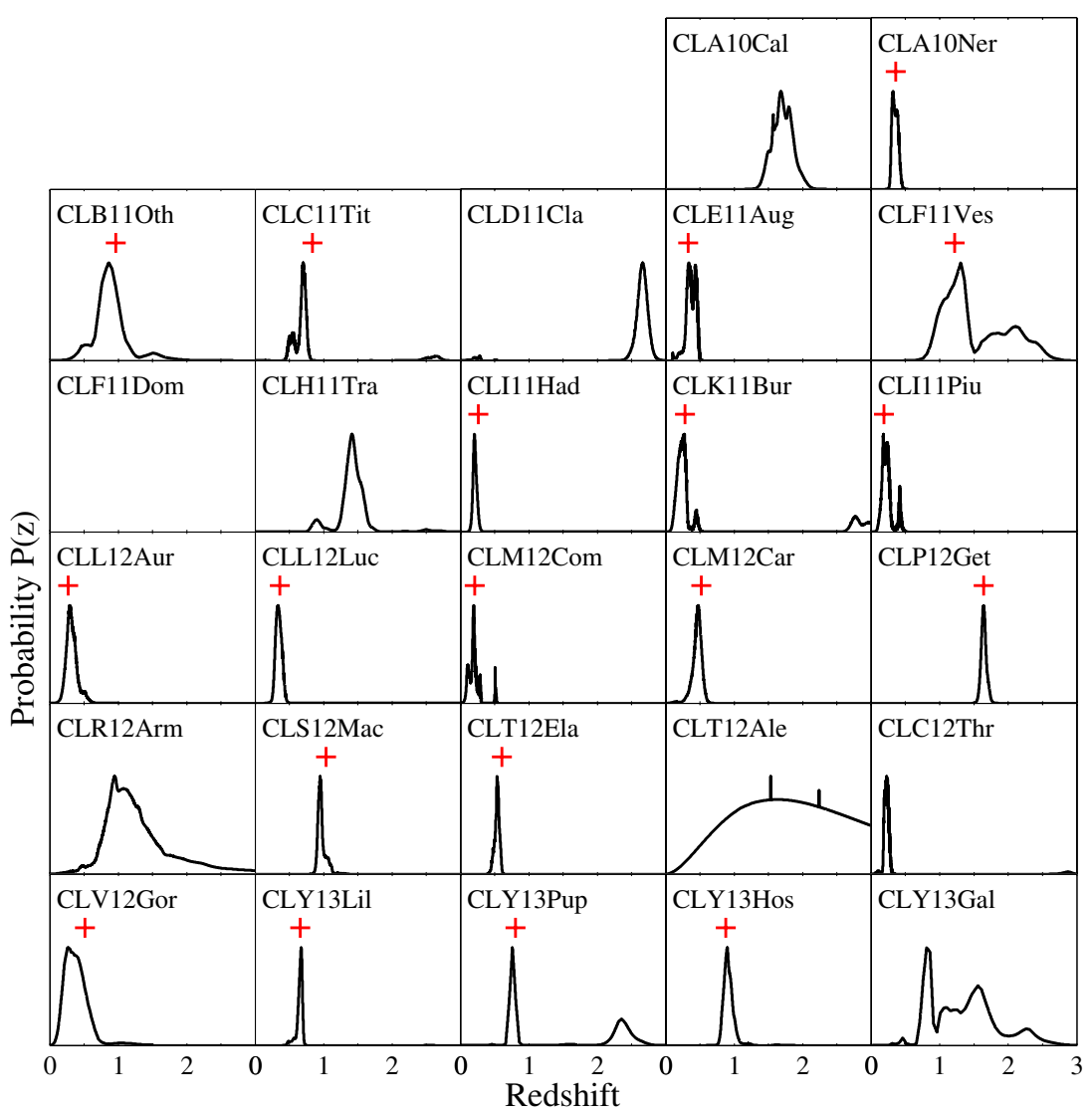

Figure 5. BPZ $z$-PDFs of the SN host galaxies. The $z$-PDFs are the solid curves and the spectroscopic redshifts, where available, are marked by red crosses. The designation of each SN appears in the upper left corner of each panel. All $z$-PDFs have been normalized so that $\int P(z) d z=1$. CLF11Dom is a "hostless" SN (see Section 3.5).

(A color version of this figure is available in the online journal.)

below in Section 4, when deriving the $\mathrm{SN}$ Ia rates, we sum the $P$ (Ia) values of all the $\mathrm{SNe}$ in our sample

The apparent magnitudes of each SN are measured with aperture photometry on the subtraction images of each epoch using the IRAF routine apphot and the same apertures described in Section 2.1. The zero-point magnitudes and aperture corrections for ACS filters are taken from Sirianni et al. (2005). For WFC3-IR and WFC3-UVIS, we use the zero-point magnitudes calculated for a 0'.4 aperture, as of 2012 March 6, by the Space Telescope Science Institute. ${ }^{25}$ The aperture corrections for the WFC3 filters were calculated by measuring the photometry of several bright stars using different apertures and adopting the correction for a 0.4 aperture.

For the SN Ia simulations, we use the Guy et al. (2007) SALT2 $^{26}$ model, with nuisance parameters for the redshift, stretch $(x 1)$, color $(c)$, and time of peak brightness. The corecollapse (CC) SNe are generated from the SNANA library of 43 CC SN templates, taken from the SN samples of the SDSS (Frieman et al. 2008; Sako et al. 2008; D'Andrea et al. 2010), Supernova Legacy Survey (SNLS; Astier et al. 2006), and Carnegie Supernova Project (Hamuy et al. 2006; Stritzinger et al. 2009; Morrell 2012). Each of these CC SN models also has parameters for redshift, host extinction $\left(A_{V}\right)$, date of peak brightness, and peak luminosity.

\footnotetext{
$25 \mathrm{http} / / /$ www.stsci.edu/hst/wfc3/phot_zp_lbn

26 http://supernovae.in2p3.fr/ guy/salt/
}

The remainder of our technique is fundamentally similar to other Bayesian light-curve classifiers (e.g., Kuznetsova \& Connolly 2007; Poznanski et al. 2007a; Rodney \& Tonry 2009; Sako et al. 2011): we compute the likelihood that a given model matches the observable data, multiply it by priors for the model parameters, then marginalize over all models to derive the final posterior classification probability.

The simulated $\mathrm{SNe}$ are reddened and dimmed according to the Cardelli et al. (1989) reddening law and one of three hostgalaxy dust extinction models: "low," "medium," and "high" dust models. For the simulated SNe Ia, the "low" dust model is the Barbary et al. (2012) skewed Gaussian fit to the Astier et al. (2006) SNLS, while the "mid" and "high" dust models are taken from Kessler et al. (2009a) and Neill et al. (2006), respectively. For $\mathrm{CC} \mathrm{SNe,} \mathrm{we} \mathrm{use} \mathrm{models} \mathrm{composed} \mathrm{of} \mathrm{a} \mathrm{half}$ Gaussian centered at $A_{V}=0 \mathrm{mag}$ and an exponential of the form $e^{-\left(A_{V} / \tau_{V}\right)}$. These models have three parameters: the standard distribution of the Gaussian, $\sigma_{A_{V}}$; the characteristic $A_{V}$ value, $\tau_{V}$; and the ratio between the Gaussian and power-law components at $A_{V}=0, A_{0}$. For the "low," "mid," and "high" dust models, the values of these parameters are $\sigma_{A_{V}}=0.15,0.6$, and $0.5 \mathrm{mag} ; \tau_{V}=0.5,1.7$, and 2.8; and $A_{0}=1$, 4, and 3 mag.

The peak magnitudes of each $\mathrm{SN}$ subtype are chosen according to their observed luminosity functions (LFs), which are detailed in Table 3. The Li et al. (2011b) LFs, derived from a local sample of SNe observed by the Lick Observatory Supernova Search (LOSS; Leaman et al. 2011; Li et al. 2011a, 2011b), were not originally corrected for host-galaxy extinction. Here, 

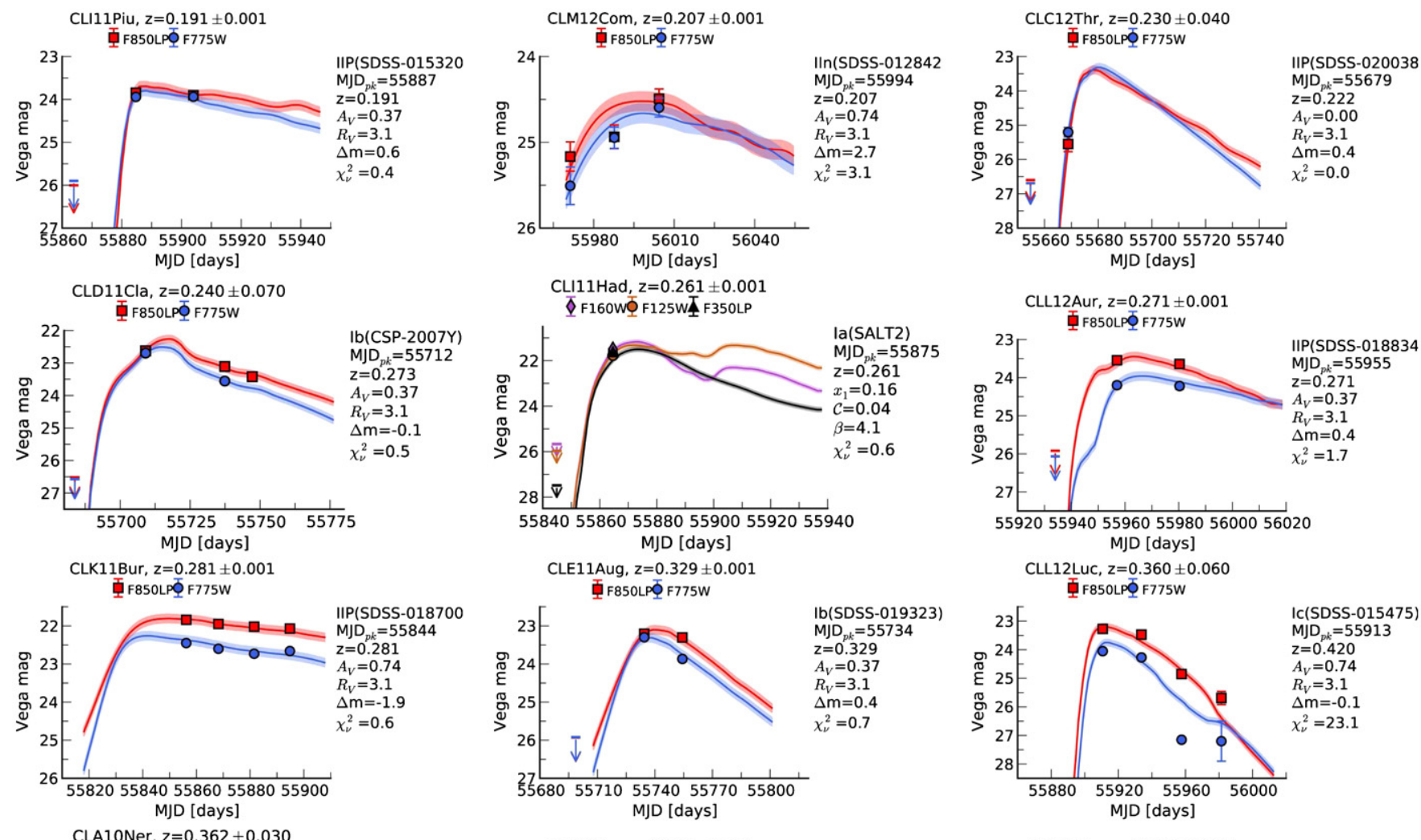

Ib(SDSS-019323)

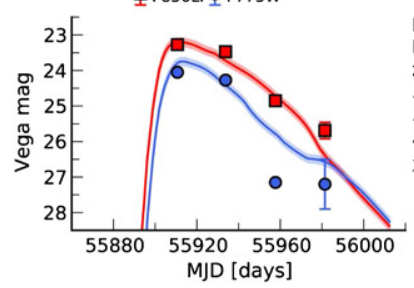

IC(SDSS-015475) $\mathrm{MJD}_{p k}=55734$

$\mathrm{Z}=0.329$

$A_{V}=0.37$

$R_{V}=3.1$

$\Delta \mathrm{m}=0.4$
$\chi_{\nu}^{2}=0.7$

CLA10Ner, $z=0.362 \pm 0.030$ 百F850LP页F775W

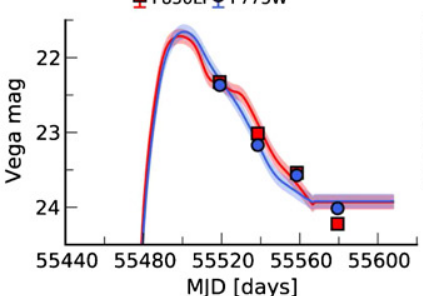

la(SALT2) $\mathrm{MJD}_{p k}=55499$ $\mathrm{z}=0.361$ $x_{1}=-1.11$ $x_{1}=-1.11$ $\mathcal{C}=-0.03$ $\beta=4.1$
$\chi_{\nu}^{2}=3.8$

CLF11Dom, $z=0.700 \pm 0.100$
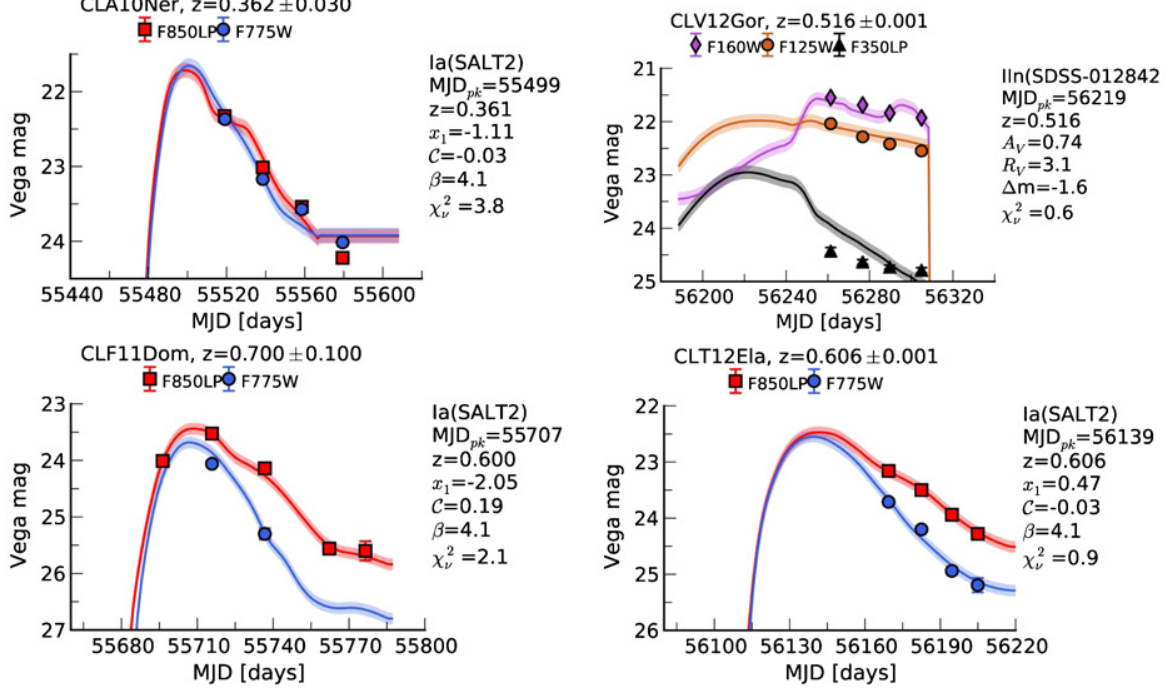

CLM12Car, $z=0.518 \pm 0.001$

百F850LP页F775W

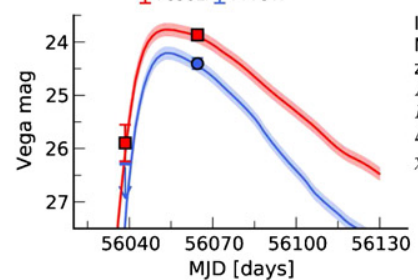

Ic(SDSS-015475) $\mathrm{MJD}_{p k}=56054$ $\mathrm{z}=0.518$ $A_{V}=0.37$ $R_{V}=3.1$ $\Delta \mathrm{m}=0.1$ $\chi_{\nu}^{2}=1.7$
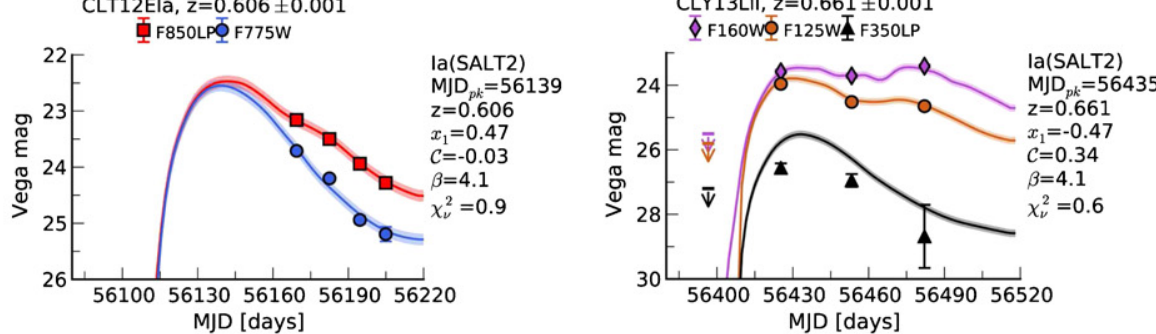

Figure 6. Best-fitting STARDUST light curves for 15 of the $27 \mathrm{SN}$ candidates. Each panel shows the multi-band photometry and best-fitting template light curve of the maximum-likelihood SN type. The title of each panel gives the SN name and its prior redshift, along with a legend detailing the different filters used for photometry. The table to the right of the light curve details the maximum-probability SN template; the posterior values of the light-curve fit parameters, including the date of peak brightness, redshift, and color and shape parameters; and the reduced $\chi^{2}$ value of the fit, $\chi_{v}^{2}$. Downturned arrows denote $3 \sigma$ upper limits on the flux.

(A color version of this figure is available in the online journal.)

we adopt "dust-free" LFs for SNe II-P and II-L such that when applying the "medium" dust model, the resultant simulated LFs approximate those published by Li et al. (2011b).

A further prior is placed on the fraction of each SN type as a function of redshift. The distribution of SN type fractions has only been measured in the local universe ( $\mathrm{Li}$ et al. 2011b), and it is expected to change with increasing redshift, once the SN Ia rate starts to deviate from the star-formation rate. However, assuming a prior on the evolution of the SN type fraction requires us to assume a prior on the CC SN and SN Ia rates as a function of redshift. Such a prior might bias the SN Ia rates measured in this work, and so we classify our
SN sample twice: once using this prior, and once assuming that the fraction of SN types remains constant with redshift. While the latter assumption is probably not the case in reality, it expresses our lack of concrete knowledge on the subject. We report the classification probability, $P(\mathrm{Ia})$, of each $\mathrm{SN}$ in our sample in Table 2 both with $\left(P(\mathrm{Ia})_{w p}\right)$ and without $\left(P(\mathrm{Ia})_{n p}\right)$ the SN type fraction prior. The uncertainty reported for each $P(\mathrm{Ia})_{w p}$ value takes into account uncertainties in both the extinction and SN-fraction priors, while the uncertainty of $P(\mathrm{Ia})_{n p}$ reflects only the uncertainty in the extinction prior. For each of the $\mathrm{SNe}$ in our sample, the resultant $P$ (Ia) values are consistent with each other, and while generally $P(\mathrm{Ia})_{w p}<P(\mathrm{Ia})_{n p}$, the difference between 

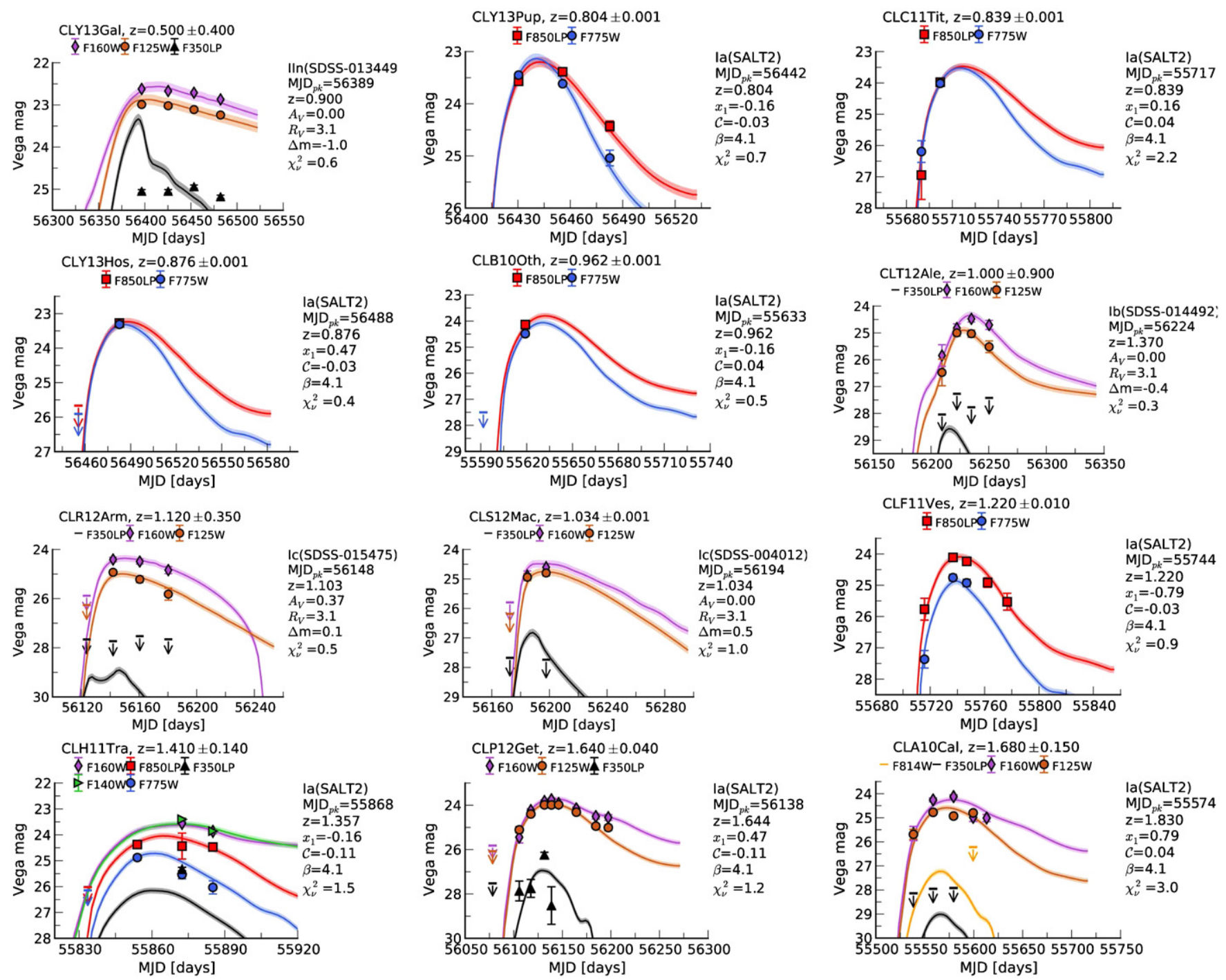

Figure 7. Figure 6, continued, showing light-curve fits for the remaining $12 \mathrm{SN}$ candidates.

(A color version of this figure is available in the online journal.)

Table 3

SN Luminosity Functions Used for SN Classification

\begin{tabular}{lccl}
\hline \hline Type & $M_{R}$ & $\sigma$ & \multicolumn{1}{c}{ Source } \\
\hline Ia & -19.37 & 0.47 & Wang et al. (2006) \\
Ib & -17.90 & 0.90 & Drout et al. (2011) \\
Ic & -18.30 & 0.60 & Drout et al. (2011) \\
IcBL & -19.00 & 1.10 & Drout et al. (2011) \\
II-P & -16.56 & 0.80 & Li et al. (2011b) \\
II-L & -17.66 & 0.42 & Li et al. (2011b) \\
IIn & -18.25 & 1.00 & Kiewe et al. (2012) \\
\hline
\end{tabular}

Notes. The Li et al. (2011b) LFs have been corrected for host-galaxy extinction, as detailed in the text.

the two values is small and has a negligible effect on the final SN Ia rates. The resultant light-curve fits, obtained without the $\mathrm{SN}$-fraction prior, are presented in Figures 6 and 7.

$\mathrm{SNe}$ that were caught on the rise and were suspected of being SNe Ia at $z>1$ were followed up with further HST imaging in order to follow the evolution of their light curves. In our sample, these include CLA10Cal, CLF11Ves, CLH11Tra, CLP12Get, CLR12Arm, and CLT12Ale. Three SNe were caught sufficiently early, and were bright enough, to be followed up spectroscopically, either from the ground or with HST. These were CLI11Had, CLF11Ves, and CLY13Pup, whose spectra were obtained with Keck+LRIS, the ACS G800L grism, and VLT+FORS2, respectively. Using the Supernova Identification code (SNID ${ }^{27}$; Blondin \& Tonry 2007), we classify CLI11Had and CLY13Pup as SNe Ia having the spec- $z$ measured from each of their host galaxies. The best-fitting SNID templates are overlaid on the SN spectra in Figure 2. Owing to its high redshift, the ACS G800L grisms caught CLF11Ves only in the rest-frame range $\sim 2500-4500 \AA$, and SNID fails to classify the spectrum as belonging to any type of $\mathrm{SN}$ at $z=1.22$. When allowed to fit for the SN redshift, SNID classifies CLF11Ves as either a SN $\mathrm{Ia}$ or $\mathrm{SN} \mathrm{Ib}$ at $z \approx 0.95$. Consequently, we do not claim to have spectroscopic confirmation for CLF11Ves as a SN Ia, although in the bottom panel of Figure 2, we show that the spectrum could be fit with the Hsiao et al. (2007) SN Ia template at peak and at $z=1.22$.

Because declining $\mathrm{SNe}$ appear in all four epochs, their photometry cannot be measured from the subtraction images.

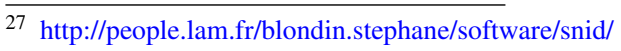


CLA10Ner, CLF11Dom, and CLL12Luc are well offset from their respective host galaxies, so we measure their photometry from the target images and assume any contamination by galactic light is minimal. CLT12Ela is in a relatively faint (in the observed bands) area of its host galaxy. Here, too, we assume that any contamination by galactic light is minimal. As can be seen in Figure 3, CLK11Bur, CLV12Gor, and CLY13Gal exploded in relatively bright regions of their host galaxies, so contamination is a certainty. However, all three of these $\mathrm{SNe}$ show signs of either a plateau or a slow decline in their light curves, and are classified as SNe II, as shown in Figures 6 and 7.

\subsection{Notes on Individual Supernovae}

We make the following more detailed notes on a number of cases with ambiguous host identification, redshift, or classification.

CLD11Cla has a broad $z$ probability density function (PDF) with a prominent peak at $z=2.66_{-0.09}^{+0.07}$ and a secondary peak at $z=0.24_{-0.04}^{+0.07}$. We have attempted to obtain spectra of the host galaxy of this SN using several instruments (VLT+FORS2, Keck+LRIS, and the ACS+G800L grism). However, the host galaxy appears to have an absorption spectrum with no discernible emission lines suitable for determining its redshift. At $z \approx 2.7$, the $\mathrm{SN}$ would be too bright to be either a SN Ia or a CC SN. While it could still be a high- $z$ superluminous SN (Gal-Yam 2012), it is more likely that this is a normal CC SN at $z=0.24$, at which its colors agree with those of CC SNe simulated using SNANA. In any case, we exclude this $\mathrm{SN}$ from the SN Ia sample.

CLF11Dom was discovered on the decline, but actually peaked during the period of the survey, as evidenced by its photometry. It has no immediately discernible host galaxy, the closest lying $\sim 4^{\prime \prime} .7$ away, with a photo- $z$ of $0.71_{-0.09}^{+0.12}$. When classifying this $\mathrm{SN}$, we chose a wide redshift prior of $0.7 \pm 0.6$ and found that the light curve was best fit as belonging to a $\mathrm{SN}$ Ia at $z \approx 0.6$, consistent with the photo- $z$ of the nearby galaxy.

CLL12Luc is a declining SN with four possible host galaxies. Of those, three have photo- $z$ values of $0.33,0.59$, and 1.13 . One has a spec- $z$ of 0.36 that is consistent with its photo- $z$ of 0.33 . At these redshifts, the $\mathrm{SN}$ would be $\sim 45,30$, and $70 \mathrm{kpc}$ away from the core of each galaxy, respectively. Based on the colors of the SN, and assuming it peaked 40-70 days before the first observation epoch, it could either be a CC SN at $z=0.36$ or a SN Ia at $z \approx 0.6$. However, the SN colors measured from the last two observation epochs are inconsistent with the SN Ia solution, so in this work we treat this $\mathrm{SN}$ as a CC SN.

Like CLL12Luc, CLC12Thr has several potential host galaxies, for three of which we have measured the photo- $z$. Two galaxies, a face-on spiral and an elliptical, are at $z=0.23$, while the third is an E/S0 galaxy at $z \approx 1.6$. At either redshift, the colors of the SN are consistent with those of a CC SN. However, the $z=0.23$ spiral and elliptical galaxies are much closer to the $\mathrm{SN}$ than the $z \approx 1.6$ galaxy: $\sim 60,30$, and $155 \mathrm{kpc}$, respectively. Based on these data, we classify this SN as a CC SN at $z=0.23$. It would be interesting to inspect the elliptical galaxy, which is closer to the $\mathrm{SN}$, for evidence of recent star formation.

We have discovered four SNe Ia at $z>1.2$ : CLA10Cal, CLF11Ves, CLH11Tra, and CLP12Get. Although we have not yet succeeded in obtaining the spectroscopic redshift of the host galaxy of CLA10Cal, its photo- $z$ is based on both Subaru data and HST NIR photometry in the F125W and F160W bands, so we are confident of its redshift. CLP12Get has a secure redshift from a photo- $z$ fit based on nine bands from the optical
(Subaru) to the NIR (HST). The spectrum of its host galaxy, obtained with VLT $+\mathrm{X}$-shooter, shows no emission or absorption lines, but the photometry extracted from its continuum is consistent with the photo- $z$ fit. CLF11Ves, as noted above, has a secure, spectroscopic redshift of 1.22. All four candidates have $P(\text { Ia })_{w p, n p}>0.95$.

There are, at most, three more SNe with broad photo- $z$ PDFs that could potentially enter this redshift range: CLR12Arm, CLT12Ale, and CLY13Gal. However, all three SNe are classified as $\mathrm{CC}$ SNe according to their light curves, with posterior redshifts of $\sim 1.1,1.4$, and 0.9 , respectively. CLT12Ale exploded in a faint host galaxy $(\mathrm{F} 160 \mathrm{~W}=26.4 \pm 0.1 \mathrm{mag})$. Based on the available Subaru photometry and additional HST photometry in F125W and F160W, the photo- $z$ of the galaxy can only be constrained to the very wide range $0.5-3.0$. When considering the two-parameter space spanned by the host galaxy's redshift and type, we find that the most likely redshift solutions are either at $z \approx 1$ or $z>5$. Although superluminous $\mathrm{SNe}$ have been observed out to redshift $\sim 4$, they are exceedingly rare (Cooke et al. 2012). It follows that the more likely redshift solution for this host galaxy is $\sim 1$. This is strengthened by the light-curve classification of this $\mathrm{SN}$, which finds a posterior redshift of $\sim 1.4$. As there is some probability that CLT12Ale is a SN Ia $\left(P(\mathrm{Ia})_{n p}=0.13 \pm 0.10\right)$, we include it in our calculation of the SN Ia rates, as detailed in Section 4, below.

\section{THE TYPE-Ia SUPERNOVA RATE}

In this section, we use the SN Ia sample from Section 3, along with the detection efficiencies as a function of magnitude measured in Section 3.2 and the classification probabilities measured in Section 3.4, to measure the rates of SNe Ia as a function of redshift, or lookback time. So as not to bias our results, we use the $\mathrm{SN}$ classification without the assumption of the SN-fraction prior.

The SNe Ia in our sample can be divided among three categories, according to when they reached maximum light: before, during, or after the monitored interval of time spent on each field. Each category will have a distinct detection efficiency as a function of redshift. The date of maximum light can occur up to 40 days before and 20 days after the duration of the survey. These values were chosen according to the approximate time when the SNe Ia in our sample reached their peak, relative to the survey times in the fields where they were discovered, based on preliminary light-curve fits. Accordingly, the visibility time of our survey is defined as the sum of the times each parallel field in each cluster was monitored (i.e., the time between the first and last epoch of that field), with the addition of 40 days before and 20 days after the observation period, in order to account for the $\mathrm{SNe}$ Ia in our sample that were caught either in decline or on the rise. Adding more or less time to the survey-extension times will either raise or lower, respectively, the number of $\mathrm{SNe}$ included in the rate calculation. To within Poisson errors, the change in extension time should, in principle, cancel out the change in SN numbers, leaving the resultant SN Ia rate unchanged.

We define the rate, $R_{\mathrm{Ia}}$, in a redshift bin bound by redshifts $z_{1}$ and $z_{2}$, as

$$
R_{\mathrm{Ia}}\left(z_{1}<z<z_{2}\right)=\frac{\sum_{i} N_{i}(z) / \eta_{i}(z)}{\sum_{j} t_{j} A_{j} \int_{z_{1}}^{z_{2}} \frac{1}{(1+z)} \frac{d V}{d z} d z},
$$

where $N_{i}$ is the number of $\mathrm{SNe}$ Ia (see below); $\eta_{i}$ is that category's detection efficiency at the redshift, $z$, of each SN; 
$t_{j}$ is the visibility time, composed of the time between the first and last epoch of observation of a field $j$, plus 40 days before the start of the survey and 20 days after its end; $A_{j}$ is the solid angle of the searchable area of field $j$, divided by $4 \pi$ steradians; $d V$ are thin volume elements behind each searchable area; and the $(1+z)$ factor converts the rates from the observer frame to the rest frame. Although we classify a SN as a SN Ia if $P(\mathrm{Ia}) \geqslant 0.5$, we define $N_{i}$ as the sum of $P(\mathrm{Ia})_{n p}$ values of all the $\mathrm{SNe}$ in each subcategory (before, during, or after the monitored interval). This is based on our treatment of $P(\mathrm{Ia})$ as a measure of the probability of a SN being a SN Ia. Thus, for example, CLD11Cla has a $P(\mathrm{Ia})_{n p}=19 \%$ probability of being a SN Ia, and is counted accordingly. This approach allows us to take into account the uncertainty of our classifications, especially for $\mathrm{SNe}$ with sparse data.

To compute the detection efficiency of each SN category, $\eta$, we must first convert our detection efficiency from a function of magnitude to a function of redshift. We do this by using the measured detection efficiency as a function of magnitude from Section 3.2 to simulate the discovery process of $\sim 25,000$ SNe Ia. For each SN, we use the Hsiao et al. (2007) SN Ia spectral templates to simulate a light curve. The Hsiao et al. (2007) spectral templates are normalized so that an unredshifted, "normal" (i.e., with stretch $s=1$ ) SN Ia at maximum light has a $B$-band apparent magnitude of $M_{B}=0$. These templates are first redshifted and reddened using the Cardelli et al. (1989) extinction law. We perform synthetic photometry on the redshifted and reddened spectral templates in the survey filters (F850LP and F160W) and construct light curves according to Perlmutter et al. (1999):

$$
m=m_{z, s=1}+M_{B}+\mu-\alpha(s-1),
$$

and

$$
t_{s, z}=t_{z=0, s=1} \alpha(1+z),
$$

where $m_{z, s=1}$ is the apparent magnitude of a "normal" SN Ia with $s=1$ at redshift $z ; M_{B}$ is the absolute magnitude of the $\mathrm{SN}$ in the $B$ band at maximum light; $\mu$ is the distance modulus at redshift $z ; \alpha=1.52 \pm 0.14$ (Astier et al. 2006); and $t_{s, z}$ is the time axis of the light curve, stretched as a result of the stretch, $s$, and time dilation at redshift $z$.

Each $\mathrm{SN}$ is assigned a random cluster and field (WFC3 or ACS), redshift, $M_{B}$, stretch value, and host-galaxy extinction value $\left(A_{V}\right)$. The redshift values are drawn from a flat distribution in the range $0-3$. Following $\mathrm{G} 11$, the absolute $B$-band magnitude at maximum light is drawn from a Gaussian centered on $M_{B}=-19.37$ with a standard deviation of $\sigma_{M_{B}}=0.17$ (this standard deviation, smaller than the one used for the SN Ia LF as it appears in Table 3, reflects the spread in SN Ia intrinsic luminosity after correcting for the luminosity-stretch relation; Hamuy et al. 1995, 1996; Riess et al. 1996; Phillips et al. 1999). The stretch values, following Sullivan et al. (2006), are drawn from a Gaussian centered on $s=1$ with a standard deviation of $\sigma_{s}=0.25$ and limited to the range $0.6<s<1.4$. This distribution is wide enough to account for both subluminous and overluminous SNe Ia. The distribution of the amount of dust in the vicinities of SNe Ia is as yet poorly constrained. To gauge the systematic uncertainty of the SN Ia rate caused by this, as in Section 3.4 above, we follow D08 and Barbary et al. (2012) and use four different host-galaxy extinction models in our simulation: Hatano et al. (1998), Riello \& Patat (2005), Neill et al. (2006), and Kessler et al. (2009a), which have average extinctions of $\left\langle A_{V}\right\rangle=0.3,0.3,0.4$, and $0.2 \mathrm{mag}$,

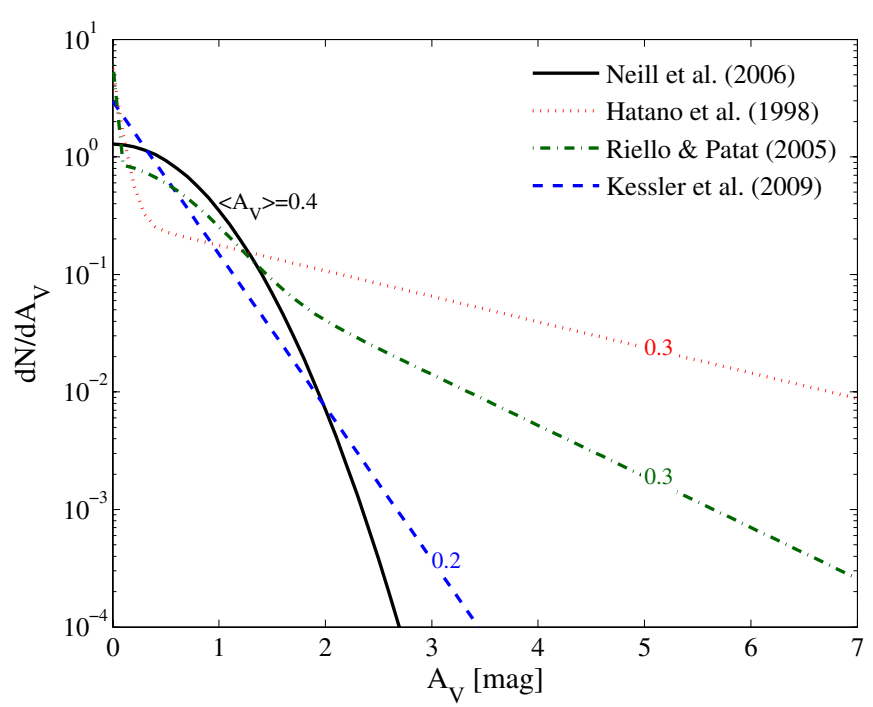

Figure 8. SN Ia host-galaxy dust extinction models used in the derivation of the SN Ia rates. We use the Neill et al. (2006) model (solid black curve) as the fiducial model. While the models shown here go out to $A_{V}=7 \mathrm{mag}$, we use values only to $A_{V}=3 \mathrm{mag}$, as nearly all the objects produced by these models fall in the range $A_{V}=0-3 \mathrm{mag}$.

(A color version of this figure is available in the online journal.)

respectively. To remain consistent with G11, we choose the Neill et al. (2006) model as our fiducial host-galaxy extinction model. These models are illustrated in Figure 8. Although we show these models (and specifically Hatano et al. 1998 and Riello \& Patat 2005) going out to $A_{V}=7 \mathrm{mag}$, we use values only out to $A_{V}=3 \mathrm{mag}$, after which all models produce a negligible number of objects.

After choosing when the SN reached peak, the light curve is sampled according to the survey cadence in that particular cluster and field, and subtraction magnitudes are computed by subtracting the flux in each search epoch from the flux of the reference image. Using the detection efficiency at the resultant magnitude in each search epoch, the $\mathrm{SN}$ is either discovered or missed. The resultant detection-efficiency curves for SNe Ia that reached maximum light before, during, or after the monitored interval are shown in Figure 9.

We divide the $\mathrm{SNe}$ in our sample into three redshift bins: $0<z<0.6,0.6<z<1.2$, and $1.2<z<1.8$. In each redshift bin, we compute the effective redshift, $z_{\text {eff }}$, as

$$
z_{\mathrm{eff}}\left(z_{1}<z<z_{2}\right)=\frac{\int_{z_{1}}^{z_{2}} \frac{z}{(1+z) \eta(z)} d V}{\int_{z_{1}}^{z_{2}} \frac{1}{(1+z) \eta(z)} d V}
$$

In each redshift bin, we take the minimal and maximal differences between the rate as computed with the fiducial dust model and with the models in Figure 8 as lower and upper systematic uncertainties owing to dust extinction. Since we express the number of SNe Ia in our final sample as the sum of all the SN $P(\mathrm{Ia})_{n p}$ values, we also propagate the uncertainties in these values and add to the rates a systematic uncertainty due to our classification technique. The systematic uncertainties from dust extinction and classification are then summed. G11 also considered the systematic uncertainty due to the expected increase in extinction as a result of dust at high redshifts (e.g., Mannucci et al. 2007; Mattila et al. 2012). However, G11 did not take into account the different extinction models used here. Specifically, the Hatano et al. (1998) extinction model adds $\mathrm{a} \sim+9 \%$ systematic uncertainty to the $\mathrm{SN}$ Ia rate in 


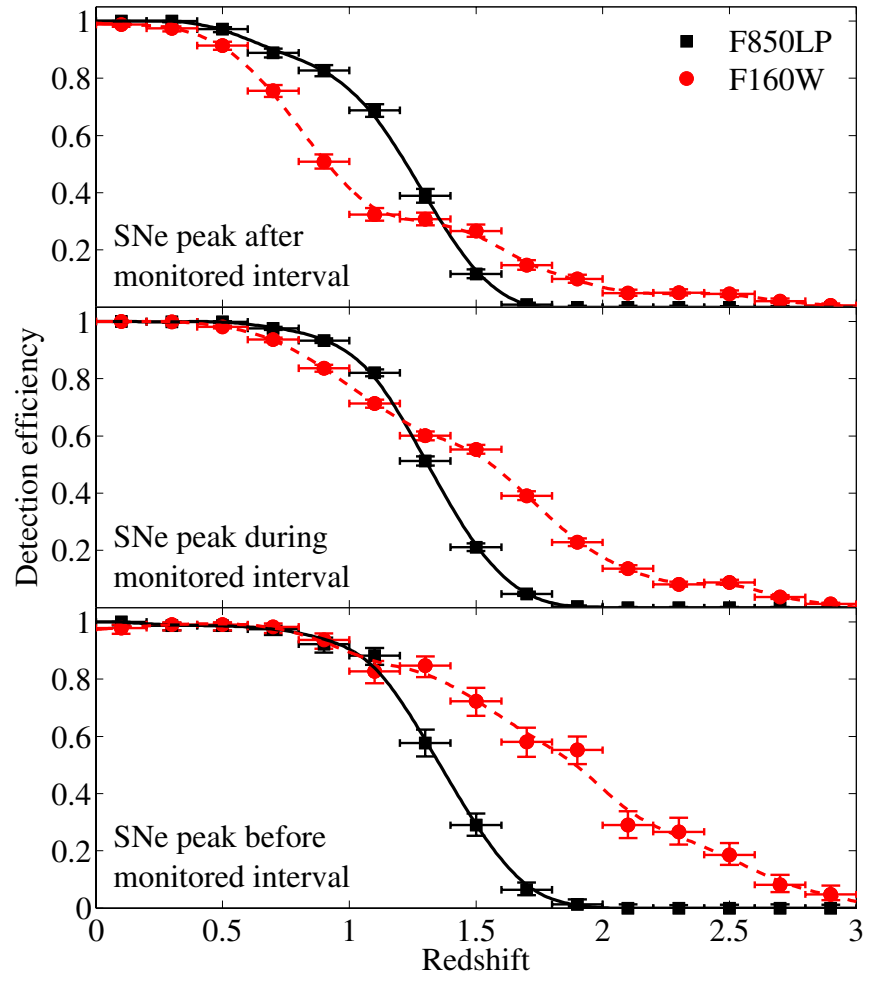

Figure 9. SN Ia detection efficiency as a function of redshift in the F850LP (solid black) and F160W (dashed red) bands for SNe Ia that reached maximum light in the $B$ band before (bottom), during (center), and after (top) the monitored interval.

(A color version of this figure is available in the online journal.)

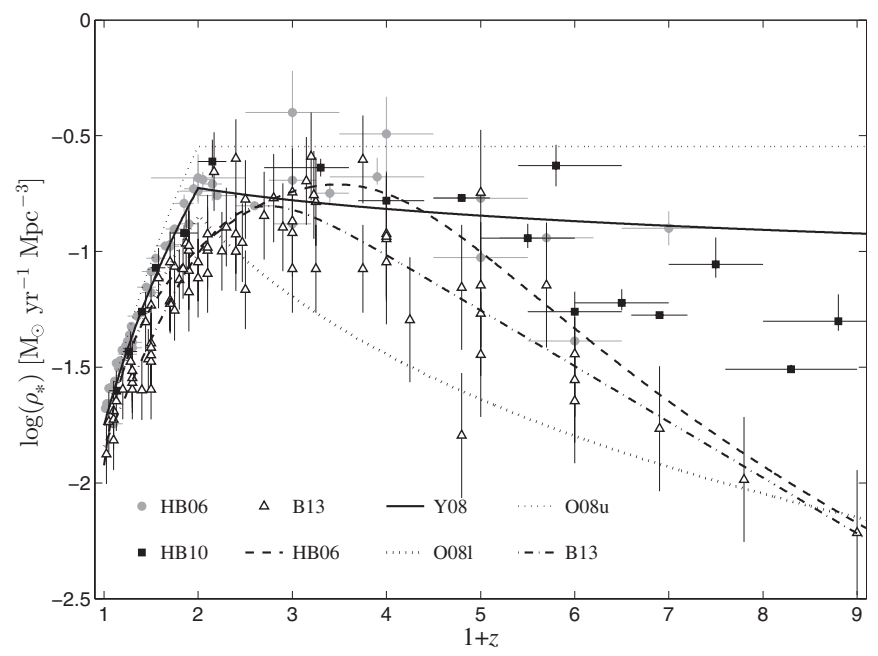

Figure 10. SFH measurements and parameterizations. SFH measurement compilations up to 2006 from HB06 are shown in filled circles, and additional measurements up to 2010 compiled by Horiuchi \& Beacom (2010), here marked as HB10, are shown as filled squares. The most up-to-date compilation from B13 is shown as open triangles. The different parameterizations are shown as curves and include the Cole et al. (2001) fit to the HB06 data (dashed); the Y08 (solid) and $\mathrm{O} 08 \mathrm{u} / \mathrm{O} 081$ (thin/thick dotted) power-law fits; and the B13 parameterization (dot-dashed). All data and parameterizations have been rescaled to the Bell et al. (2003) "diet" Salpeter IMF.

the $1.2<z<1.8$ bin, similar to the $\sim+10 \%$ systematic uncertainty G11 added to their SN Ia rate at $1.5<z<2.0$.

While we found no $\mathrm{SNe}$ at $z>1.8$, Figure 9 shows that WFC3 is still sensitive to SNe Ia out to $z \approx 2.5$. Consequently, we add a fourth redshift bin, $1.8<z<2.4$, and compute

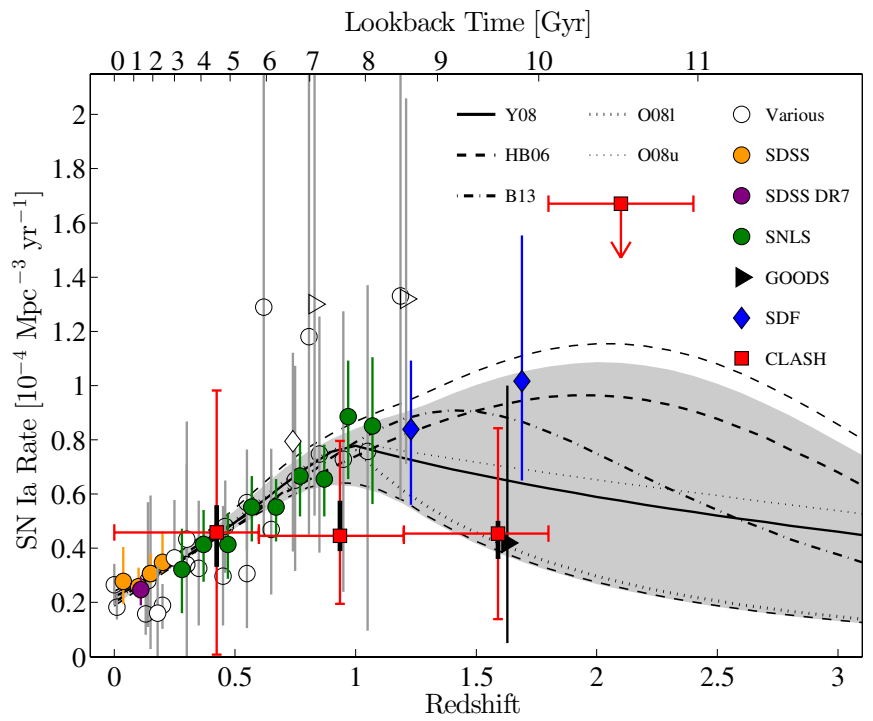

Figure 11. SN Ia rates from CLASH (filled, red squares) compared to rates from the literature and best-fitting SN Ia rate evolutions derived by convolving a power-law DTD with different SFHs. Circles denote data from surveys with measurements out to $z \approx 1$ from Cappellaro et al. (1999), Hardin et al. (2000), Pain et al. (2002), Tonry et al. (2003), Blanc et al. (2004), Botticella et al. (2008), Horesh et al. (2008), Rodney \& Tonry (2010), Li et al. (2011a), Barbary et al. (2012), and Melinder et al. (2012). Filled circles denote the most accurate and precise measurements at $z<1$ and are from the SDSS Stripe 82 survey (Dilday et al. 2010; orange), SNLS (Perrett et al. 2012; green), and SDSS DR7 (Graur \& Maoz 2013; purple). The GOODS rates from Dahlen et al. (2008) are shown as triangles and the SDF rates from Graur et al. (2011) are shown as diamonds. The $z>1.5$ rates from these two surveys are colored in black and blue, respectively. The thick curves are convolutions of several SFHs (dashed, Hopkins \& Beacom 2006; solid, Yüksel et al. 2008; thin/thick dotted, Oda et al. 2008; dot-dashed, Behroozi et al. 2013) with the best-fitting power-law DTDs. The shaded area is the confidence region resulting from the combined $68 \%$ statistical uncertainties in the values of the power-law index fit with the above SFHs. The thin dashed lines indicate the $68 \%$ statistical uncertainty region obtained without the new CLASH measurements. All vertical error bars are sums of the statistical and systematic uncertainties. The CLASH vertical error bars are composed of the systematic uncertainty, shown as black thick lines, and the statistical uncertainty, shown as red thin lines. The horizontal error bars delineate the CLASH redshift bins. The Perrett et al. (2012) and $z>1.5$ Dahlen et al. (2008) SN Ia rates have been shifted by $\Delta z=+0.02$ to disentangle them from other results.

(A color version of this figure is available in the online journal.)

a $2 \sigma$ upper limit to the SN Ia rate in that bin by taking the $95 \%$ Poisson uncertainty in the number of SNe found in the bin (zero), and considering the detection efficiency of the different $\mathrm{SN}$ categories in the center of the bin at $z=2.1$.

The resultant $\mathrm{SN}$ Ia rates, including both statistical and systematic uncertainties, are shown in Figure 11. Table 4 summarizes the SN Ia rates, with and without correction for host-galaxy dust extinction, and Table 5 shows the complete error budget of our SN Ia rates. Table 6 compares the rates from this work to previous rates from the literature. Where necessary, the measurements have been corrected to reflect the value of $h=0.7$ used in this work. As Perrett et al. (2012) did not take into account low-stretch, SN1991bg-like SNe Ia, we scale up their SN Ia rates by $15 \%$ (see their Section 6). As in G11, in instances where rates were originally reported in units of $\mathrm{SNuB}$ (SNe per century per $10^{10} L_{\odot, B}$; Cappellaro et al. 1999; Hardin et al. 2000; Pain et al. 2002; Madgwick et al. 2003; Blanc et al. 2004), we have converted them to volumetric rates using the Botticella et al. (2008) redshift-dependent luminosity density function,

$$
j_{B}(z)=(1.03+1.76 z) \times 10^{8} L_{\odot, B} \mathrm{Mpc}^{-3} .
$$


Table 4

SN Ia Numbers and Rates

\begin{tabular}{|c|c|c|c|c|}
\hline Subsample & $0.0<z<0.6$ & $0.6<z<1.2$ & $1.2<z<1.8$ & $1.8<z<2.4$ \\
\hline Total & 12 & 11 & 4 & 0 \\
\hline SN host galaxies with spec- $z$ & 10 & 7 & 2 & 0 \\
\hline Hostless $\mathrm{SNe}$ & 0 & 1 & 0 & 0 \\
\hline SNe Ia (raw) & 2.4 & 6.4 & 4.1 & 0 \\
\hline SNe Ia (efficiency-corrected) & 2.4 & 7.0 & 8.0 & 0 \\
\hline SN Ia rate without host-galaxy extinction ${ }^{a}$ & $0.45_{-0.32,-0.13}^{+0.42,+0.10}$ & $0.42_{-0.18,-0.05}^{+0.21,+0.12}$ & $0.27_{-0.13,-0.06}^{+0.21,+0.03}$ & $<0.6$ \\
\hline SN Ia rate $\left(10^{-4} \mathrm{yr}^{-1} \mathrm{Mpc}^{-3}\right)$ & $0.46_{-0.32,-0.13}^{+0.42,+0.10}$ & $0.45_{-0.19,-0.06}^{+0.22,+0.13}$ & $0.45_{-0.22,-0.09}^{+0.34,+0.05}$ & $<1.7$ \\
\hline Effective redshift & 0.42 & 0.94 & 1.59 & 2.1 \\
\hline
\end{tabular}

Notes. The $1.8<z<2.4$ rate is a $2 \sigma$ upper limit.

a The errors, separated by commas, are respectively the $68 \%$ Poisson statistical uncertainties on the number of SNe, and systematic uncertainties due to possible misclassification and different host-galaxy extinction models, respectively.

Table 5

SN Ia Rate Uncertainty Percentages

\begin{tabular}{lccc}
\hline \hline Uncertainty & $0<z<0.6$ & $0.6<z<1.2$ & $1.2<z<1.8$ \\
\hline Poisson & $+92 /-71$ & $+49 /-44$ & $+75 /-49$ \\
Classification & $+17 /-28$ & $+4.6 /-9.4$ & $+1.9 /-2.7$ \\
Dust model & $+5.4 /-0.1$ & $+25 /-3.3$ & $+8.6 /-18$ \\
Total & $+114.4 /-99.1$ & $+78.6 /-56.7$ & $+85.5 /-69.7$ \\
\hline
\end{tabular}

Note. All uncertainties are reported as percentage of the rates.

\section{THE TYPE-Ia SUPERNOVA DELAY-TIME DISTRIBUTION}

In this section, we test different models of the DTD by convolving them with various cosmic SFHs and fitting the resultant SN Ia rate histories to the SN Ia rate measurements from the previous section, along with rates from the literature. We include all the rate measurements from Table 6 except for Neill et al. (2006, 2007), which have been superseded by Perrett et al. (2012); Dahlen et al. (2004) and Kuznetsova et al. (2008), which have been superseded by D08; Barris \& Tonry (2006), which has been superseded by Rodney \& Tonry (2010); Poznanski et al. (2007b), which has been superseded by G11; and Madgwick et al. (2003) and Krughoff et al. (2011), which have been superseded by Graur \& Maoz (2013). We do not use the $z>2$ upper limit from the previous section as it is too high to affect the DTD fits. In total, we use $50 \mathrm{SN}$ Ia rate measurements, of which 41 are at $z<1$ and 9 are at $z>1$.

Following G11, we test different SFHs, including the Cole et al. (2001) parameterization fit to the data collected by Hopkins \& Beacom (2006, HB06); the SFH presented by Yüksel et al. (2008, Y08) and upper (O08u) and lower (O081) limits from Oda et al. $(2008,008)$ which can be approximated as broken power laws with a break at $z=1$ and with varying indices before and after the break; and the recent Behroozi et al. (2013, B13) SFH. These SFHs, and the data they are based on, are presented in Figure 10.

When deriving SFH measurements, various authors use different versions of the initial-mass function (IMF), leading to different scalings of the SFH. In order to maintain consistency across the different SFHs, we must choose one IMF and rescale the SFHs accordingly. As in G11 and Graur \& Maoz (2013), we assume a "diet" Salpeter IMF (Bell et al. 2003), which is similar to the Salpeter (1955) IMF with lower and upper mass limits of 0.1 and $125 M_{\odot}$, respectively, but with a stellar mass-to-light $(M / L)$ ratio that is scaled down by a factor of 0.7 in order to fit the $M / L$ ratios measured in disks (Bell \& de Jong 2001). The choice of this IMF requires us to scale down the SFHs of HB06, O08, and Y08, who assumed a Salpeter (1955) IMF, by a factor of 0.7. The B13 SFH, where a Chabrier (2003) IMF was assumed, is scaled up by a factor of 0.7 . To allow comparisons with G11, we use the Y08 SFH as the fiducial model in our DTD recoveries and the other SFHs to estimate a systematic uncertainty in the values of the parameters of the DTD model tested below.

We test a power-law DTD of the form $\Psi(t)=$ $\Psi_{1 \mathrm{Gyr}}(t / 1 \mathrm{Gyr})^{\beta}$, setting its index, $\beta$, and scaling, $\Psi_{1 \mathrm{Gyr}}$, as free parameters, leaving 48 degrees of freedom for the fit. The DTD is set to zero before $40 \mathrm{Myr}$, to allow for $8 M_{\odot}$ stars to evolve into CO WDs. The Y08 SFH yields a bestfit index value of $\beta=-1.00_{-0.06(0.10)}^{+0.06(0.09)}$ with a reduced $\chi^{2}$ $\left(\chi_{v}^{2}\right)$ of 0.7 , where the statistical uncertainties are the $68 \%$ and 95\% (in parentheses) confidence regions, respectively. The other SFHs yield a systematic uncertainty of ${ }_{-0.08}^{+0.12}$, with $\chi_{v}^{2}$ values in the range $0.7-0.8$, yielding a final value of $\beta=$

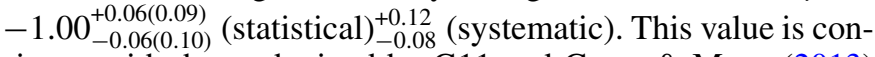
sistent with those obtained by G11 and Graur \& Maoz (2013) and in a variety of different SN surveys and using different DTD recovery techniques (see Maoz \& Mannucci 2012). Integrating the DTD over a Hubble time, we find that the number of SNe Ia per formed mass, $N / M_{*}$, lies in the range $(0.5-1.3) \times 10^{-3} \mathrm{SNe} M_{\odot}^{-1}$, similar to the ranges found by G11 and Graur \& Maoz (2013). The best-fitting SN Ia rate histories derived from each SFH, along with the $68 \%$ uncertainty region, are shown in Figure 11.

We investigate also the viability of a Gaussian DTD fit (Strolger et al. 2004, 2010) to the SN Ia rates. We start by testing the Gaussian DTD proposed by D08, with a mean delay time of $3.4 \mathrm{Gyr}$ and a standard deviation of $0.68 \mathrm{Gyr}$. As in G11, we allow the scaling of this DTD to vary as a free parameter. Coupled with the SN Ia rates, the only SFH that does not disfavor this DTD is the HB06 SFH, with a reduced $\chi^{2}$ value of 1.1. All other SFHs result in SN Ia rate evolutions that are excluded at a $>95 \%$ significance level, with the $\mathrm{O} 08$ and B13 SFHs specifically excluded at a $>99 \%$ significance level. We next test a general Gaussian DTD, where we allow the mean delay time, standard deviation, and scaling to vary as free parameters, while requiring that $95 \%$ of the area under the DTD remain above a delay time of $40 \mathrm{Myr}$ (thus ensuring the resultant DTD retains a Gaussian shape). The B13 and lower-limit O08 SFHs result in Gaussians that are excluded at a $>95 \%$ significance level. 
Table 6

SN Ia Rate Measurements

\begin{tabular}{|c|c|c|c|c|c|c|c|}
\hline Redshift & $N_{\text {Ia }}$ & Rate $\left(10^{-4} \mathrm{yr}^{-1} \mathrm{Mpc}^{-3}\right)$ & Reference & Redshift & $N_{\text {Ia }}$ & Rate $\left(10^{-4} \mathrm{yr}^{-1} \mathrm{Mpc}^{-3}\right)$ & Reference \\
\hline 0.01 & 70 & $0.183 \pm 0.046$ & Cappellaro et al. (1999) ${ }^{\mathrm{a}}$ & 0.55 & 72 & $0.55_{-0.07,-0.06}^{+0.07,+0.05}$ & Perrett et al. $(2012)^{\mathrm{e}}$ \\
\hline$<0.019$ & 274 & $0.265_{-0.033,-0.043}^{+0.034,+0.043}$ & Li et al. $(2011 \mathrm{a})^{\mathrm{b}}$ & 0.552 & 41 & $0.63_{-0.10,-0.27}^{+0.10,+0.26}$ & Neill et al. $(2007)^{\mathrm{d}}$ \\
\hline 0.0375 & 516 & $0.278_{-0.083,-0.000}^{+0.112,+0.015}$ & Dilday et al. $(2010)^{\mathrm{c}}$ & 0.62 & 7 & $\begin{array}{l}1.29_{-0.57,-0.28}^{+0.88,+0.27} \\
-0.20\end{array}$ & Melinder et al. (2012) \\
\hline 0.09 & 17 & $0.29_{-0.07}^{+0.09}$ & Dilday et al. (2008) & 0.65 & 23 & $1.49 \pm 0.31$ & Barris \& Tonry (2006) ${ }^{\mathrm{d}}$ \\
\hline 0.098 & 19 & $0.24_{-0.12}^{+0.12}$ & Madgwick et al. $(2003)^{\mathrm{a}, \mathrm{d}}$ & 0.65 & 10.09 & $0.49_{-0.17,-0.08}^{+0.17,+0.14}$ & Rodney \& Tonry (2010) \\
\hline 0.1 & 516 & $0.259_{-0.044,-0.001}^{+0.052,+0.018}$ & Dilday et al. $(2010)^{\mathrm{c}}$ & 0.65 & 91 & $0.55_{-0.06,-0.07}^{+0.06,+0.05}$ & Perrett et al. $(2012)^{\mathrm{e}}$ \\
\hline 0.1 & 52 & $0.569_{-0.085,-0.047}^{+0.098,+0.058}$ & Krughoff et al. (2011) ${ }^{\mathrm{d}}$ & 0.714 & 42 & $1.13_{-0.19,-0.70}^{+0.19,+0.54}$ & Neill et al. $(2007)^{\mathrm{d}}$ \\
\hline 0.11 & 90 & $0.247_{-0.026,-0.031}^{+0.029,+0.016}$ & Graur \& Maoz (2013) & 0.74 & 5.5 & $0.43_{-0.32}^{+0.36}$ & Poznanski et al. (2007b) ${ }^{\mathrm{d}}$ \\
\hline 0.13 & 14 & $0.158_{-0.043,-0.035}^{+0.056,+0.035}$ & Blanc et al. (2004) ${ }^{\mathrm{a}}$ & 0.74 & 20.3 & $0.79_{-0.41}^{+0.33}$ & Graur et al. (2011) \\
\hline 0.14 & 4 & $0.28_{-0.13,-0.04}^{+0.22,+0.07}$ & Hardin et al. $(2000)^{\mathrm{a}}$ & 0.75 & 28 & $1.78 \pm 0.34$ & Barris \& Tonry $(2006)^{\mathrm{d}}$ \\
\hline 0.15 & 516 & $0.307_{-0.034,-0.005}^{+0.038,+0.035}$ & Dilday et al. $(2010)^{\mathrm{c}}$ & 0.75 & 14.29 & $0.68_{-0.21,-0.14}^{+0.21,+0.23}$ & Rodney \& Tonry (2010) \\
\hline 0.15 & 1.95 & $0.32_{-0.23,-0.06}^{+0.23,+0.07}$ & Rodney \& Tonry (2010) & 0.75 & 110 & $0.67_{-0.07,-0.08}^{+0.07,+0.06}$ & Perrett et al. $(2012)^{\mathrm{e}}$ \\
\hline 0.16 & 4 & $0.16_{-0.10,-0.14}^{+0.10,+0.07}$ & Perrett et al. $(2012)^{\mathrm{e}}$ & 0.80 & 14 & $1.57_{-0.25,-0.53}^{+0.44,+0.75}$ & Dahlen et al. $(2004)^{\mathrm{d}}$ \\
\hline 0.2 & 17 & $0.189_{-0.034,-0.015}^{+0.042,+0.018} \pm 0.42$ & Horesh et al. (2008) & 0.80 & 18.33 & $0.93_{-0.25}^{+0.25}$ & Kuznetsova et al. $(2008)^{\mathrm{d}}$ \\
\hline 0.2 & 516 & $0.348_{-0.030,-0.007}^{+0.032,+0.082}$ & Dilday et al. $(2010)^{\mathrm{c}}$ & 0.807 & 5.25 & $1.18_{-0.45,-0.28}^{+0.60,+0.44}$ & Barbary et al. (2012) \\
\hline 0.25 & 1 & $0.17 \pm 0.17$ & Barris \& Tonry (2006) ${ }^{\mathrm{d}}$ & 0.83 & 25 & $1.30_{-0.27,-0.51}^{+0.33,+0.73}$ & Dahlen et al. (2008) \\
\hline 0.25 & 516 & $0.365_{-0.028,-0.012}^{+0.031,+0.182}$ & Dilday et al. $(2010)^{\mathrm{c}}$ & 0.85 & 15.43 & $0.78_{-0.22,-0.16}^{+0.22,+0.31}$ & Rodney \& Tonry (2010) \\
\hline 0.26 & 16 & $0.32_{-0.08,-0.08}^{+0.08,+0.07}$ & Perrett et al. $(2012)^{\mathrm{e}}$ & 0.85 & 128 & $0.66_{-0.06,-0.08}^{+0.06,+0.07}$ & Perrett et al. $(2012)^{\mathrm{e}}$ \\
\hline 0.3 & 31.05 & $0.34_{-0.15,-0.22}^{+0.16,+0.21}$ & Botticella et al. $(2008)^{\mathrm{f}}$ & 0.94 & 6.4 & $0.45_{-0.19,-0.06}^{+0.22,+0.13}$ & CLASH (this work) \\
\hline 0.3 & 516 & $0.434_{-0.034,-0.016}^{+0.037,+0.396}$ & Dilday et al. $(2010)^{\mathrm{c}}$ & 0.95 & 13.21 & $0.76_{-0.25,-0.26}^{+0.25,+0.32}$ & Rodney \& Tonry (2010) \\
\hline 0.35 & 5 & $0.530 \pm 0.024$ & Barris \& Tonry (2006) ${ }^{\mathrm{d}}$ & 0.95 & 141 & $0.89_{-0.09,-0.14}^{+0.09,+0.12}$ & Perrett et al. $(2012)^{\mathrm{e}}$ \\
\hline 0.35 & 4.01 & $0.34_{-0.19,-0.03}^{+0.19,+0.07}$ & Rodney \& Tonry (2010) & 1.05 & 11.01 & $0.79_{-0.28,-0.41}^{0.28,+0.36}$ & Rodney \& Tonry (2010) \\
\hline 0.35 & 31 & $0.41_{-0.07,-0.07}^{+0.07,+0.06}$ & Perrett et al. $(2012)^{\mathrm{e}}$ & 1.05 & 50 & $0.85_{-0.14,-0.15}^{+0.14,+0.12}$ & Perrett et al. $(2012)^{\mathrm{e}}$ \\
\hline 0.368 & 17 & $0.31_{-0.05,-0.03}^{+0.05,+0.08}$ & Neill et al. $(2007)^{\mathrm{d}}$ & 1.187 & 5.63 & $1.33_{-0.49,-0.26}^{+0.65,+0.69}$ & Barbary et al. (2012) \\
\hline 0.40 & 3 & $0.69_{-0.27,-0.25}^{+0.34,+1.54}$ & Dahlen et al. $(2004)^{\mathrm{d}}$ & 1.20 & 6 & $1.15_{-0.26,-0.44}^{+0.47,+0.32}$ & Dahlen et al. $(2004)^{\mathrm{d}}$ \\
\hline 0.40 & 5.44 & $0.53_{-0.17}^{+0.39}$ & Kuznetsova et al. (2008) ${ }^{\mathrm{d}}$ & 1.20 & 8.87 & $0.75_{-0.30}^{+0.35}$ & Kuznetsova et al. $(2008)^{\mathrm{d}}$ \\
\hline 0.42 & 2.4 & $0.46_{-0.32,-0.13}^{+0.42,+0.10}$ & CLASH (this work) & 1.21 & 20 & $1.32_{-0.29,-0.32}^{+0.36,+0.38}$ & Dahlen et al. (2008) \\
\hline 0.442 & 0 & $0.00_{-0.00,-0.00}^{+0.50,+0.00}$ & Barbary et al. (2012) & 1.23 & 10.0 & $1.05_{-0.56}^{+0.45}$ & Poznanski et al. $(2007 \mathrm{~b})^{\mathrm{d}}$ \\
\hline 0.45 & 9 & $0.73 \pm 0.24$ & Barris \& Tonry $(2006)^{\mathrm{d}}$ & 1.23 & 27.0 & $0.84_{-0.28}^{+0.25}$ & Graur et al. (2011) \\
\hline 0.45 & 5.11 & $0.31_{-0.15,-0.04}^{+0.15,+0.12}$ & Rodney \& Tonry (2010) & 1.535 & 1.12 & $0.77_{-0.54,-0.77}^{+1.07,+0.44}$ & Barbary et al. (2012) \\
\hline 0.45 & 42 & $0.41_{-0.07,-0.06}^{+0.07,+0.05}$ & Perrett et al. $(2012)^{\mathrm{e}}$ & 1.55 & 0.35 & $0.12_{-0.12}^{+0.58}$ & Kuznetsova et al. (2008) ${ }^{\mathrm{d}}$ \\
\hline 0.46 & 8 & $0.48 \pm 0.17$ & Tonry et al. (2003) & 1.59 & 4.1 & $\begin{array}{l}0.45_{-0.22,-0.09}^{+0.34,+0.05} \\
-0.00\end{array}$ & CLASH (this work) \\
\hline 0.467 & 73 & $0.42_{-0.06,-0.09}^{+0.06,+0.13}$ & Neill et al. $(2006)^{d}$ & 1.60 & 2 & $0.44_{-0.25,-0.11}^{+0.32,+0.14}$ & Dahlen et al. $(2004)^{\mathrm{d}}$ \\
\hline 0.47 & 8 & $0.80_{-0.27,-0.26}^{+0.37,+1.66}$ & Dahlen et al. (2008) & 1.61 & 3 & $0.42_{-0.23,-0.14}^{+0.39,+0.19}$ & Dahlen et al. (2008) \\
\hline 0.55 & 38 & $0.568_{-0.088,-0.088}^{+0.098,+0.098}$ & Pain et al. (2002) ${ }^{\mathrm{a}}$ & 1.67 & 3.0 & $0.81_{-0.60}^{+0.79}$ & Poznanski et al. $(2007 b)^{\mathrm{d}}$ \\
\hline 0.55 & 29 & $2.04 \pm 0.38$ & Barris \& Tonry $(2006)^{\mathrm{d}}$ & 1.69 & 10.0 & $1.02_{-0.37}^{+0.54}$ & Graur et al. (2011) \\
\hline 0.55 & 6.49 & $0.32_{-0.14,-0.07}^{+0.14,+0.07}$ & Rodney \& Tonry (2010) & 2.1 & $\mathbf{0}$ & $<1.7$ & CLASH (this work) ${ }^{\mathrm{g}}$ \\
\hline
\end{tabular}

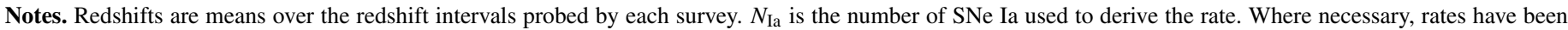
converted to $h=0.7$. Where reported, the statistical errors are followed by systematic errors, and separated by commas. Rates from this work are shown in bold.

${ }^{a}$ Rates have been converted to volumetric rates using Equation (7).

${ }^{\mathrm{b}} \mathrm{Li}$ et al. (2011a) consider SNe Ia within $80 \mathrm{Mpc}$.

${ }^{\mathrm{c}}$ Dilday et al. (2010) compute their rates using 516 SNe Ia at $z<0.5$.

$\mathrm{d}$ These measurements have been superseded by more recent results, as detailed in Section 5 .

e Perrett et al. (2012) do not include SN1991bg-like SNe Ia in their rates. Here, their measurements are scaled up by 15\% (see their Section 6).

${ }^{\mathrm{f}}$ Botticella et al. (2008) found a total of $86 \mathrm{SN}$ candidates of all types. See their Section 5.2 for details on their various subsamples and classification techniques.

g $2 \sigma$ upper limit on the SN Ia rate, as derived in Section 4.

The Y08, upper-limit O08, and HB06 SFHs, on the other hand, result in Gaussians with means in the range $\mu=2.7-3.3 \mathrm{Gyr}$ with standard deviations of $\sigma=0.8-1.6 \mathrm{Gyr}$ and reduced $\chi^{2}$ values of $0.9-1.3$. These Gaussian DTDs are centered at slightly lower mean delay times, but are wider, than the D08 Gaussian DTD. The resultant fits to the SN Ia rate evolution are shown in Figure 12. Although at first sight, it might appear that the $z>1.5 \mathrm{SDF}$ rate measurement is driving the exclusion of the 

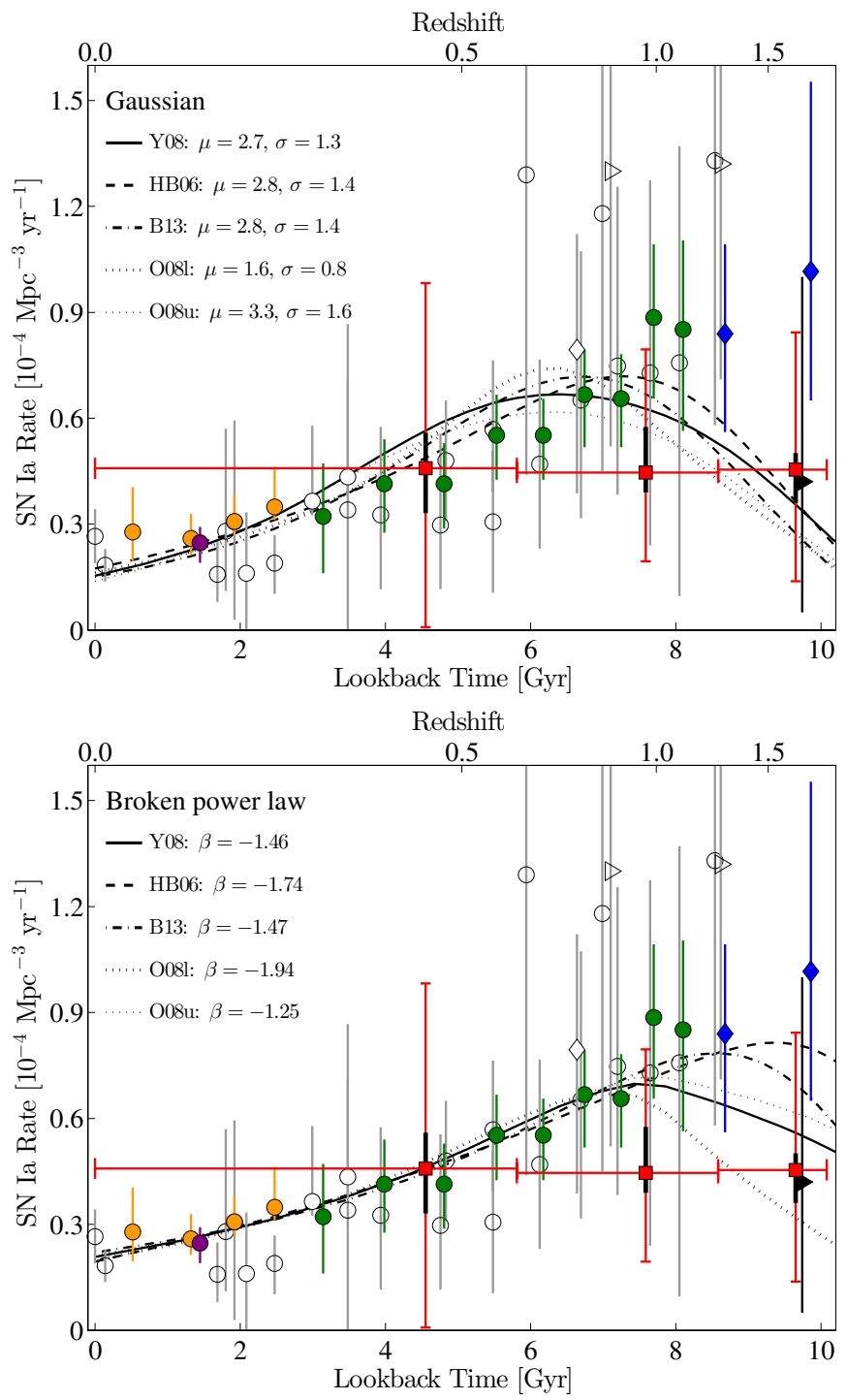

Figure 12. Observed SN Ia rates compared to predicted SN Ia rate evolution tracks from the convolution of different SFHs with a best-fitting (top) Gaussian DTD and (bottom) broken power-law DTD of the form $\Psi(t)=\Psi(t / 1 \mathrm{Gyr})^{-1 / 2}$ up to $t_{c}=1.7 \mathrm{Gyr}$, and $\Psi(t) \propto t^{\beta}$ afterward. Symbols are as in Figure 11 . The SFHs used for each fit are listed in the top panel along with best-fitting parameter values: the mean, $\mu$, and standard deviation, $\sigma$, of the Gaussian DTD, in Gyr; and the slope of the second power law, $\beta$, of the broken power-law DTD. The CLASH upper limit at $z>1.8$, which was not used in the fits, has been removed.

(A color version of this figure is available in the online journal.)

Gaussian DTDs, most of the fitting power actually comes from the accurate $z<1$ SDSS and SNLS measurements.

As in G11, we test the further possibility that at early times the DTD is dominated by the production efficiency of double WD systems, which is described by a power law of the form $t^{-0.5}$ (Pritchet et al. 2008), until some cutoff time, $t_{c}$, when a second physical process takes over, described as a power law having a different slope, $t^{\beta}$. Whereas in G11, we set the slope of the second power law to $\beta=-1$ and fit for $t_{c}$, here we set $t_{c}=1.7$ Gyr, the lifetime of a $2 M_{\odot}$ star, the least massive star expected to produce a $\sim 0.7 M_{\odot}$ CO WD (see Figure 4 of Girardi et al. 2000), and find that the best-fitting slope of the second power law is $\beta=-1.46_{-0.13(0.22)}^{+0.16(0.26)}$ (statistical) ${ }_{-0.21}^{+0.48}$ (systematic) with reduced $\chi^{2}$ values in the range $0.7-0.9$.
Finally, we test both DD and SD DTDs resulting from binary population synthesis (BPS) simulations. Here, we use updated versions of the scaled models presented in Figures 2 and 3 of Nelemans et al. (2013; for color versions of these DTD figures see Wang \& Han 2012; updated versions of the models courtesy of G. Nelemans 2013, private communication). As in Nelemans et al. (2013), we designate the BPS DTD models by the groups that computed them: Yungelson (e.g., Yungelson 2010), the Yunnan group (Wang/Han et al.; e.g., Wang et al. 2010), the StarTrack code (Ruiter et al.; e.g., Ruiter et al. 2009), the Brussels group (Mennekens et al.; e.g., Mennekens et al. 2010), the Utrecht group (Claeys et al.; e.g., Claeys et al. 2013), and the SeBa code (Bours/Toonen; e.g., Toonen et al. 2012). We present the updated versions of the scaled BPS DTD models in Figure 13. As Nelemans et al. (2013) scaled the different DTDs to a Kroupa et al. (1993) IMF, we rescale them to the diet Salpeter IMF by multiplying them by a factor of 0.7 . For comparison with the volumetric SN Ia rates, we convolve the different BPS DTDs with the B13 SFH, as parameterized by their Equation (F1), which we reproduce here as

$$
\operatorname{CSFR}(z)=\frac{C}{10^{A\left(z-z_{0}\right)}+10^{B\left(z-z_{0}\right)}}
$$

where $\operatorname{CSFR}(z)$ is the cosmic SFH as a function of redshift, and the constants $A, B, C$, and $z_{0}$ are given in Table 7 of B13 as $A=-0.997, B=0.241, C=0.180$, and $z_{0}=1.243$.

As has been commented elsewhere (e.g., Maoz \& Mannucci 2012; Nelemans et al. 2013), the BPS DTDs, both for DD and SD scenarios, fail to produce the number of observed SNe Ia. Here, we test the shape of the BPS DTDs by treating their scaling as a free parameter. The resultant SN Ia rate evolutions are presented in Figure 14. The BPS DD models require scalings by small factors of 3-9 and result in reduced $\chi^{2}$ values of $\chi_{v}^{2} \lesssim 1$, consistent with the SN Ia rates. The BPS SD models, on the other hand, require large scaling factors of $>10$ (except for the Wang/Han and Mennekens DTDs, which require scaling factors of $\sim 4$ ) and result in reduced $\chi^{2}$ values of $\chi_{v}^{2}>1.8$, thus excluding all BPS SD models at a $>99 \%$ significance level.

The poor fits of the SD models are the result of their low DTD amplitudes at long delay times. The accurate and precise $z<1$ SN Ia rates, which are most sensitive to the long delay-time component of the DTD, have the most leverage on the scalings of the BPS SD DTDs. Because the SD models have low amplitudes at long delay times, the $z<1 \mathrm{SN}$ Ia rates force scalings of large factors that then cause the resultant SN Ia rate evolutions to overshoot the $z>1$ rates.

It is instructive to compare some of the SD DTD models, and their resultant SN Ia rate evolutions, in detail. The Claeys model has the lowest amplitude at long delay times, which is why its resultant $\mathrm{SN}$ Ia rate evolution overshoots all the other models in the bottom panel of Figure 14. On the other hand, although the Yungelson model only has an intermediate delaytime component, that component is at longer delay times than the Claeys model, so it results in a SN Ia rate evolution with less amplitude than the rate evolution produced by the Claeys model. Finally, the Ruiter model has the highest amplitude at long delay times, after the Wang/Han model, which results in a low scaling. However, the Ruiter model has lower amplitude at short delay times, compared to all other DTD models besides Yungelson, which is why the low scaling, forced by the long-delay component, results in a SN Ia rate evolution that undershoots the $z>1 \mathrm{SN}$ Ia rates. 

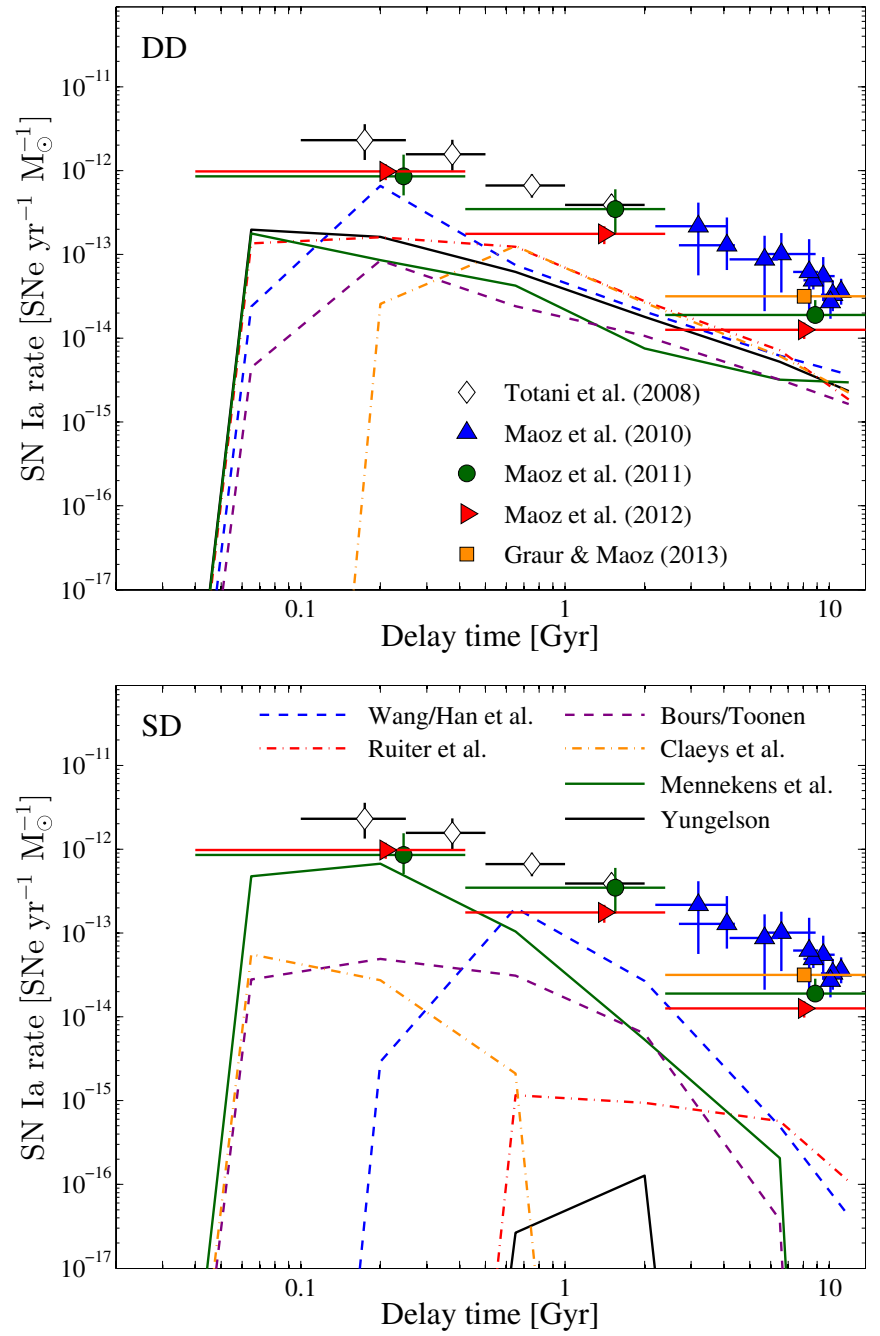

Figure 13. Binary population synthesis DTD models for the DD (top) and SD (bottom) scenarios, compared to observations. The BPS DTD models, shown here as different-colored curves, are updated versions of the ones that appear in Nelemans et al. (2013; G. Nelemans, private communication) and are listed according to the groups that computed them: Yungelson (e.g., Yungelson 2010; black solid), the Yunnan group (Wang/Han et al.; e.g., Wang et al. 2010; blue dashed), the StarTrack code (Ruiter et al.; e.g., Ruiter et al. 2009; red dot-dashed), the Brussels group (Mennekens et al.; e.g., Mennekens et al. 2010; green solid), the Utrecht group (Claeys et al.; e.g., Claeys et al. 2013; orange dot-dashed), and the SeBa code (Bours/Toonen; e.g., Toonen et al. 2012; purple dashed). For comparison, we also show reconstructed components of the DTD from observations of SNe Ia in $0.4<z<1.2$ elliptical galaxies (Totani et al. 2008; white diamonds), galaxy clusters (Maoz et al. 2010; blue triangles), LOSS-SDSS galaxies (Maoz et al. 2011; green circles), SDSS-II galaxies (Maoz et al. 2012; red right-pointing triangles), and SDSS DR7 spectra (Graur \& Maoz 2013; orange squares).

(A color version of this figure is available in the online journal.)

\section{CONCLUSIONS}

In this work, we have presented a sample of $27 \mathrm{SNe}$ discovered in the parallel fields of the 25 CLASH galaxy clusters. Of these, $\sim 13$ were classified as $\mathrm{SNe}$ Ia, four of which are at $z>1.2$. Using the SN Ia sample, we measured the $\mathrm{SN}$ Ia rate out to $z \approx 1.8$ and obtained an upper limit on the rate in the redshift range $1.8<z<2.4$. Within the uncertainties of all the measurements, these rates are consistent with both the HST/GOODS and the Subaru/SDF SN Ia rates. Based on these rates, along with previous rates from the literature, we have shown that when convolved with different cosmic SFHs, a power-law DTD with
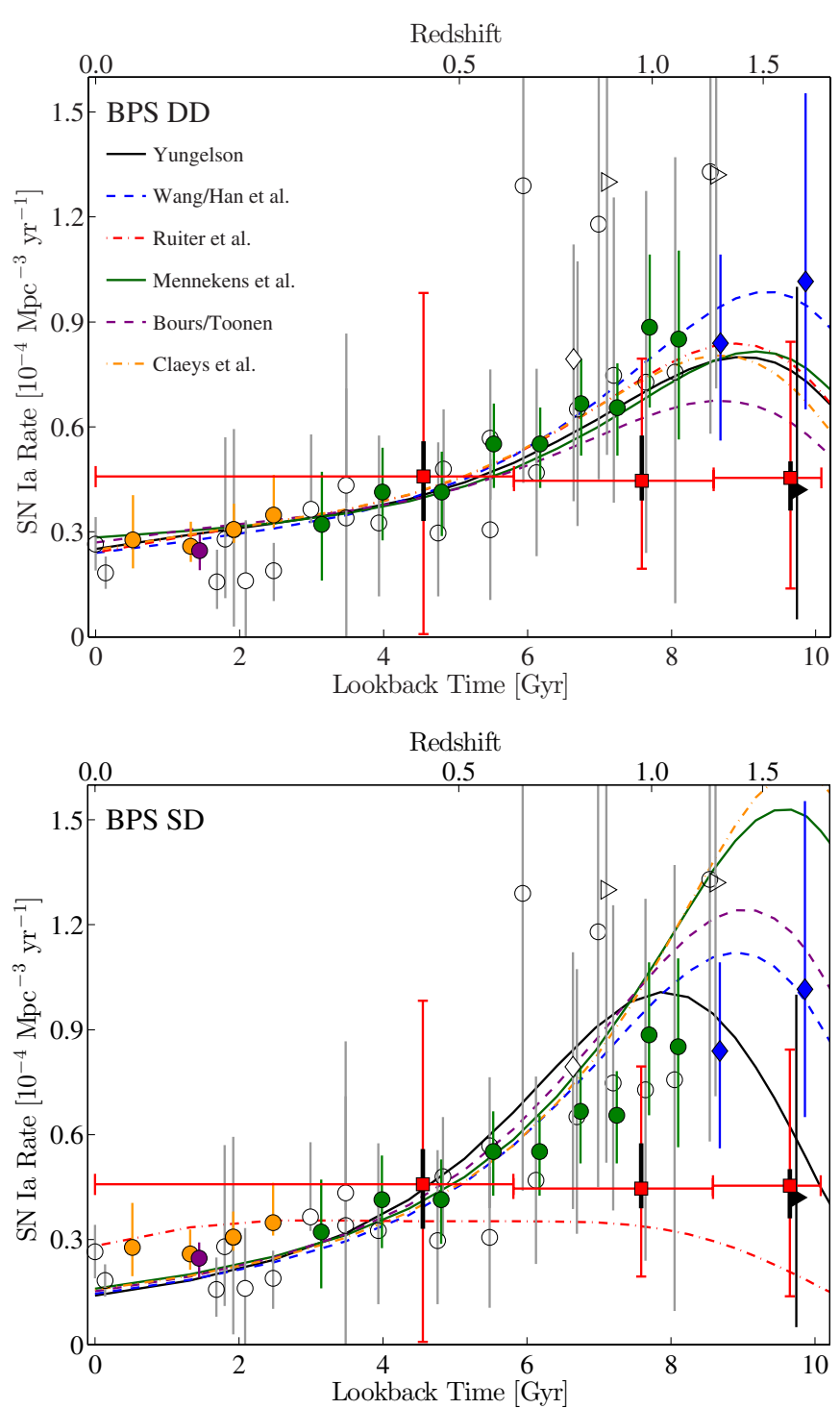

Figure 14. Observed SN Ia rates compared to predicted SN Ia rate evolution tracks from the convolution of the Behroozi et al. (2013) SFHs with BPS DD (top) and SD (bottom) DTD models from the literature (Nelemans et al. 2013). Symbols are as in Figure 12, except for the SN Ia rate evolution curves, which are labeled in the top panel according to the DTD used in each fit, as shown in Figure 13.

(A color version of this figure is available in the online journal.)

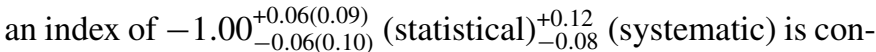
sistent with the data. The systematic uncertainty derives from the wide range of possible SFHs considered.

We have also shown that the overall shape of DTDs from BPS DD models are consistent with the SN Ia rate measurements, as long as the models are scaled up by factors of 3-9, while all BPS SD models are ruled out at a $>99 \%$ significance level.

The SN Ia rates at $z<1$ require a DTD with a significant delayed component, such as the power-law DTD tested here. The high-redshift SN Ia rates provide a probe of the early times of the DTD, where the DTD could either continue with an index of $\sim-1$, as found here, or perhaps transit to a lower index of -0.5 , as proposed by Pritchet et al. (2008). If the SD scenario contributes significantly to the SN Ia rate, as claimed by some recent work (e.g., Sternberg et al. 2011 and Dilday et al. 2012), its main effect would be on the high-redshift rates. However, to have any real discriminatory power on the different DTD 
models, the SN Ia rates at $z>1$ must be more accurate and precise than they currently are. To make the most efficient use of the CLASH SN sample, we will combine it with the final CANDELS sample in a future paper. Together, the two samples will contain a similar number of SNe Ia as the Subaru/SDF sample from G11. However, their systematic uncertainties will be lower, as they will make use of light curves and spectroscopy, where available, as done in this work. Finally, the upcoming HST Frontier Fields program ${ }^{28}$ (PI: M. Mountain) will observe six pairs of galaxy clusters and blank fields containing field galaxies, using 140 orbits of ACS and WFC3 for each pair of galaxy cluster/blank field during Cycles 21-23. Based on our work on CLASH and CANDELS, we expect that this survey, which will go deeper than either of the previous surveys, will discover $\sim 20 \mathrm{SNe}$, including five $z>1.5 \mathrm{SNe}$ Ia. Once this sample is added to the combined CLASH+CANDELS SN sample, we may finally have high-redshift SN Ia rates accurate enough to probe the early part of the DTD.

We thank Gijs Nelemans for sharing the BPS DTD models with us, and Ori Fox, Patrick Kelly, Isaac Shivvers, Brad Tucker, and WeiKang Zheng for assistance with some of the Keck observations. Financial support for this work was provided by NASA through grants HST-GO-12060 and HST-GO-12099 from the Space Telescope Science Institute (STScI), which is operated by Associated Universities for Research in Astronomy, Inc., under NASA contract NAS 5-26555. O.G. and D.M. acknowledge support by the I-CORE program of the PDC and the ISF, Grant 1829/12, and by a grant from the Israel Science Foundation. Support for S.R. was provided by NASA through Hubble Fellowship grant HF-51312.01 awarded by STScI. This work was supported by NASA Keck PI Data Awards (to Rutgers University, PI: S.W.J.), administered by the NASA Exoplanet Science Institute. Supernova research at Rutgers University is additionally supported by NSF CAREER award AST-0847157 to S.W.J. A.M. and N.B. acknowledge support from AYA201022111-C03-01 and PEX/10-CFQM-6444, and from the Spanish Ministerio de Educación y Ciencia through grant AYA200614056 BES-2007-16280. M.N. is supported by PRIN INAF2010. A.V.F. is also grateful for the support of NSF grant AST1211916, the TABASGO Foundation, and the Christopher R. Redlich Fund. The Dark Cosmology Centre is funded by the Danish National Research Foundation. J.M.S. is supported by an NSF Astronomy and Astrophysics Postdoctoral Fellowship under award AST-1302771. K.J., C.L., K.L., J.N., A.O., and H.E.R. were supported by the American Museum of Natural History's Science Research Mentoring Program under NASA grant award NNX09AL36G.

This work is based, in part, on data collected at the Subaru Telescope, which is operated by the National Astronomical Observatory of Japan. Additional data presented here were obtained at the W. M. Keck Observatory, which is operated as a scientific partnership among the California Institute of Technology, the University of California, and NASA; the Observatory was made possible by the generous financial support of the W. M. Keck Foundation. We wish to recognize and acknowledge the very significant cultural role and reverence that the summit of Mauna Kea has always had within the indigenous Hawaiian community. We are most fortunate to have the opportunity to conduct observations from this mountain.

\footnotetext{
28 http://www.stsci.edu/hst/campaigns/frontier-fields/
}

Part of the research presented here is based on observations obtained at the Gemini Observatory, which is operated by the Association of Universities for Research in Astronomy, Inc., under a cooperative agreement with the NSF on behalf of the Gemini partnership: the NSF (United States), the National Research Council (Canada), CONICYT (Chile), the Australian Research Council (Australia), Ministério da Ciência, Tecnologia e Inovaçāo (Brazil) and Ministerio de Ciencia, Tecnología e Innovación Productiva (Argentina) [Programs GS-2011A-Q-16, GN-2011A-Q-14, and GN-2012A-Q-32].

This research is based in part on observations made with ESO telescopes at the La Silla Paranal Observatory under program IDs 086.A-0660, 088.A-0708, and 089.A-0739; also, on observations collected at the European Southern Observatory, Chile (ESO Programmes 086.A-0070, 087.A-0295, 089.A-0438 and 091.A-0067).

We used data obtained with the MODS spectrographs built with funding from NSF grant AST-9987045 and the NSF Telescope System Instrumentation Program (TSIP), with additional funds from the Ohio Board of Regents and the Ohio State University Office of Research.

This research has made use of NASA's Astrophysics Data System (ADS) Bibliographic Services and of the NASA/IPAC Extragalactic Database (NED), which is operated by the Jet Propulsion Laboratory, California Institute of Technology, under contract with NASA.

Facilities: Gemini:South (GMOS-S), Gemini:Gillett (GMOSN), HST (ACS, WFC3), Keck:I (LRIS), Keck:II (DEIMOS), LBT (MODS), Subaru (Suprime-Cam), VLT:Antu (FORS2), VLT:Kueyen (X-shooter), VLT:Melipal (VIMOS)

\section{REFERENCES}

Anderson, J., \& Bedin, L. R. 2010, PASP, 122, 1035

Appenzeller, I., Fricke, K., Fürtig, W., et al. 1998, Msngr, 94, 1 Astier, P., Guy, J., Regnault, N., et al. 2006, A\&A, 447, 31

Barbary, K., Aldering, G., Amanullah, R., et al. 2012, ApJ, 745, 31

Barris, B. J., \& Tonry, J. L. 2006, ApJ, 637, 427

Behroozi, P. S., Wechsler, R. H., \& Conroy, C. 2013, ApJ, 770, 57 (B13)

Bell, E. F., \& de Jong, R. S. 2001, ApJ, 550, 212

Bell, E. F., McIntosh, D. H., Katz, N., \& Weinberg, M. D. 2003, ApJS, 149, 289

Benítez, N. 2000, ApJ, 536, 571

Bertin, E., \& Arnouts, S. 1996, A\&AS, 117, 393

Blanc, G., Afonso, C., Alard, C., et al. 2004, A\&A, 423, 881

Blondin, S., \& Tonry, J. L. 2007, ApJ, 666, 1024

Botticella, M. T., Riello, M., Cappellaro, E., et al. 2008, A\&A, 479, 49

Cappellaro, E., Evans, R., \& Turatto, M. 1999, A\&A, 351, 459

Cardelli, J. A., Clayton, G. C., \& Mathis, J. S. 1989, ApJ, 345, 245

Chabrier, G. 2003, PASP, 115, 763

Claeys, J. S. W., Pols, O. R., \& Izzard, R. G. 2013, in IAU Symp. 281, Binary Paths to Type Ia Supernovae Explosions, ed. R. Di Stefano, M. Orio, \& M. Moe (Cambridge: Cambridge Univ. Press), 236

Coe, D., Benítez, N., Sánchez, S. F., et al. 2006, AJ, 132, 926

Cole, S., Norberg, P., Baugh, C. M., et al. 2001, MNRAS, 326, 255

Cooke, J., Sullivan, M., Gal-Yam, A., et al. 2012, Natur, 491, 228

Dahlen, T., Strolger, L., \& Riess, A. G. 2008, ApJ, 681, 462 (D08)

Dahlen, T., Strolger, L.-G., Riess, A. G., et al. 2004, ApJ, 613, 189

D’Andrea, C. B., Sako, M., Dilday, B., et al. 2010, ApJ, 708, 661

Dilday, B., Howell, D. A., Cenko, S. B., et al. 2012, Sci, 337, 942

Dilday, B., Kessler, R., Frieman, J. A., et al. 2008, ApJ, 682, 262

Dilday, B., Smith, M., Bassett, B., et al. 2010, ApJ, 713, 1026

Dressel, L. 2012, Wide Field Camera 3 Instrument Handbook for Cycle 21, Version 5.0 (Baltimore, MD: STScI)

Drout, M. R., Soderberg, A. M., Gal-Yam, A., et al. 2011, ApJ, 741, 97

Faber, S. M., Phillips, A. C., Kibrick, R. I., et al. 2003, Proc. SPIE, 4841, 1657

Fioc, M., \& Rocca-Volmerange, B. 1997, A\&A, 326, 950

Förster, F., \& Schawinski, K. 2008, MNRAS, 388, L74

Frederiksen, T. F., Graur, O., Hjorth, J., Maoz, D., \& Poznanski, D. 2012, arXiv: 1211.2208

Frieman, J. A., Bassett, B., Becker, A., et al. 2008, AJ, 135, 338 
Gal-Yam, A. 2012, Sci, 337, 927

Giavalisco, M., Dickinson, M., Ferguson, H. C., et al. 2004, ApJL, 600, L103

Giavalisco, M., Sahu, K., \& Bohlin, R. C. 2002, New Estimates of the Sky Background for the HST Exposure Time Calculator, Technical Report (Baltimore, MD: STScI)

Girardi, L., Bressan, A., Bertelli, G., \& Chiosi, C. 2000, A\&AS, 141, 371

Graur, O., \& Maoz, D. 2013, MNRAS, 430, 1746

Graur, O., Poznanski, D., Maoz, D., et al. 2011, MNRAS, 417, 916 (G11)

Grazian, A., Fontana, A., de Santis, C., et al. 2006, A\&A, 449, 951

Grogin, N. A., Kocevski, D. D., Faber, S. M., et al. 2011, ApJS, 197, 35

Guy, J., Astier, P., Baumont, S., et al. 2007, A\&A, 466, 11

Hamuy, M., Folatelli, G., Morrell, N. I., et al. 2006, PASP, 118, 2

Hamuy, M., Phillips, M. M., Maza, J., et al. 1995, AJ, 109, 1

Hamuy, M., Phillips, M. M., Suntzeff, N. B., et al. 1996, AJ, 112, 2398

Hardin, D., Afonso, C., Alard, C., et al. 2000, A\&A, 362, 419

Hatano, K., Branch, D., \& Deaton, J. 1998, ApJ, 502, 177

Hildebrandt, H., Arnouts, S., Capak, P., et al. 2010, A\&A, 523, A31

Hillebrandt, W., Kromer, M., Röpke, F. K., \& Ruiter, A. J. 2013, FrPhy, 8,116

Hook, I., Allington-Smith, J. R., Beard, S. M., et al. 2003, Proc. SPIE, 4841,1645

Hopkins, A. M., \& Beacom, J. F. 2006, ApJ, 651, 142 (HB06)

Horesh, A., Poznanski, D., Ofek, E. O., \& Maoz, D. 2008, MNRAS, 389, 1871

Horiuchi, S., \& Beacom, J. F. 2010, ApJ, 723, 329

Howell, D. A. 2011, NatCo, 2, 350

Hsiao, E. Y., Conley, A., Howell, D. A., et al. 2007, ApJ, 663, 1187

Iben, I., Jr., \& Tutukov, A. V. 1984, ApJS, 54, 335

Ilbert, O., Capak, P., Salvato, M., et al. 2009, ApJ, 690, 1236

Jones, D. O., Rodney, S. A., Riess, A. G., et al. 2013, ApJ, 768, 166

Jouvel, S., Host, O., Lahav, O., et al. 2013, arXiv:1308.0063

Kashikawa, N., Shimasaku, K., Yasuda, N., et al. 2004, PASJ, 56, 1011

Kessler, R., Becker, A. C., Cinabro, D., et al. 2009a, ApJS, 185, 32

Kessler, R., Bernstein, J. P., Cinabro, D., et al. 2009b, PASP, 121, 1028

Kiewe, M., Gal-Yam, A., Arcavi, I., et al. 2012, ApJ, 744, 10

Koekemoer, A. M., Faber, S. M., Ferguson, H. C., et al. 2011, ApJS, 197, 36

Koekemoer, A. M., Fruchter, A. S., Hook, R. N., \& Hack, W. 2003, in The 2002 HST Calibration Workshop: Hubble after the Installation of the ACS and the NICMOS Cooling System, ed. S. Arribas, A. Koekemoer, \& B. Whitmore (Baltimore, MD: STScI), 337

Krist, J. E., Hook, R. N., \& Stoehr, F. 2011, Proc. SPIE, 8127, 81270J

Kroupa, P., Tout, C. A., \& Gilmore, G. 1993, MNRAS, 262, 545

Krughoff, K. S., Connolly, A. J., Frieman, J., et al. 2011, ApJ, 731, 42

Kuznetsova, N., Barbary, K., Connolly, B., et al. 2008, ApJ, 673, 981

Kuznetsova, N. V., \& Connolly, B. M. 2007, ApJ, 659, 530

Leaman, J., Li, W., Chornock, R., \& Filippenko, A. V. 2011, MNRAS, 412, 1419

Le Fèvre, O., Saisse, M., Mancini, D., et al. 2003, Proc. SPIE, 4841, 1670

Leibundgut, B. 2000, A\&ARv, 10, 179

Li, W., Chornock, R., Leaman, J., et al. 2011a, MNRAS, 412, 1473

Li, W., Leaman, J., Chornock, R., et al. 2011b, MNRAS, 412, 1441

Madgwick, D. S., Hewett, P. C., Mortlock, D. J., \& Wang, L. 2003, ApJL, 599, L33

Mannucci, F., Della Valle, M., \& Panagia, N. 2007, MNRAS, 377, 1229

Maoz, D., \& Mannucci, F. 2012, PASA, 29, 447

Maoz, D., Mannucci, F., \& Brandt, T. D. 2012, MNRAS, 426, 3282

Maoz, D., Mannucci, F., Li, W., et al. 2011, MNRAS, 412, 1508

Maoz, D., Sharon, K., \& Gal-Yam, A. 2010, ApJ, 722, 1879

Mattila, S., Dahlen, T., Efstathiou, A., et al. 2012, ApJ, 756, 111

Melinder, J., Dahlen, T., Mencía Trinchant, L., et al. 2012, A\&A, 545, A96

Mennekens, N., Vanbeveren, D., De Greve, J. P., \& De Donder, E. 2010, A\&A, 515, A89
Miyazaki, S., Komiyama, Y., Sekiguchi, M., et al. 2002, PASJ, 54, 833

Morrell, N. I. 2012, in IAU Symp. 279, Death of Massive Stars: Supernovae and Gamma-Ray Bursts, ed. P. Roming, N. Kawai, \& E. Pian (Cambridge: Cambridge Univ. Press), 361

Neill, J. D., Sullivan, M., Balam, D., et al. 2006, AJ, 132, 1126

Neill, J. D., Sullivan, M., Balam, D., et al. 2007, in AIP Conf. Ser. 924, The Multicolored Landscape of Compact Objects and Their Explosive Origins, ed. T. di Salvo, G. L. Israel, L. Piersant, L. Burderi, G. Matt, A. Tornambe, \& M. T. Menna (Melville, NY: AIP), 421

Nelemans, G., Toonen, S., \& Bours, M. 2013, in IAU Symp. 281, Binary Paths to Type Ia Supernovae Explosions, ed. R. Di Stefano, M. Orio, \& M. Moe (Cambridge: Cambridge Univ. Press), 225

Oda, T., Totani, T., Yasuda, N., et al. 2008, PASJ, 60, 169 (O08)

Oke, J. B., Cohen, J. G., Carr, M., et al. 1995, PASP, 107, 375

Pain, R., Fabbro, S., Sullivan, M., et al. 2002, ApJ, 577, 120

Perlmutter, S., Aldering, G., Goldhaber, G., et al. 1999, ApJ, 517, 565

Perrett, K., Sullivan, M., Conley, A., et al. 2012, AJ, 144, 59

Phillips, M. M., Lira, P., Suntzeff, N. B., et al. 1999, AJ, 118, 1766

Pogge, R. W., Atwood, B., Brewer, D. F., et al. 2010, Proc. SPIE, 7735, 77350A

Polletta, M., Tajer, M., Maraschi, L., et al. 2007, ApJ, 663, 81

Postman, M., Coe, D., Benítez, N., et al. 2012, ApJS, 199, 25

Poznanski, D., Maoz, D., \& Gal-Yam, A. 2007a, AJ, 134, 1285

Poznanski, D., Maoz, D., Yasuda, N., et al. 2007b, MNRAS, 382, 1169

Pritchet, C. J., Howell, D. A., \& Sullivan, M. 2008, ApJL, 683, L25

Riello, M., \& Patat, F. 2005, MNRAS, 362, 671

Riess, A. G., Filippenko, A. V., Challis, P., et al. 1998, AJ, 116, 1009

Riess, A. G., Press, W. H., \& Kirshner, R. P. 1996, ApJ, 473, 88

Riess, A. G., Strolger, L.-G., Tonry, J., et al. 2004, ApJ, 607, 665

Rodney, S. A., Riess, A. G., Strolger, L.-G., et al. 2014, arXiv:1401.7978

Rodney, S. A., \& Tonry, J. L. 2009, ApJ, 707, 1064

Rodney, S. A., \& Tonry, J. L. 2010, ApJ, 723, 47

Ruiter, A. J., Belczynski, K., \& Fryer, C. 2009, ApJ, 699, 2026

Sako, M., Bassett, B., Becker, A., et al. 2008, AJ, 135, 348

Sako, M., Bassett, B., Connolly, B., et al. 2011, ApJ, 738, 162

Salpeter, E. E. 1955, ApJ, 121, 161

Schmidt, B. P., Suntzeff, N. B., Phillips, M. M., et al. 1998, ApJ, 507, 46

Sharon, K., Gal-Yam, A., Maoz, D., et al. 2010, ApJ, 718, 876

Sirianni, M., Jee, M. J., Benítez, N., et al. 2005, PASP, 117, 1049

Sokol, J., Anderson, J., \& Smith, L. 2012, Assessing ACS/WFC Sky Backgrounds, Technical Report (Baltimore, MD: STScI)

Sternberg, A., Gal-Yam, A., Simon, J. D., et al. 2011, Sci, 333, 856

Stritzinger, M., Mazzali, P., Phillips, M. M., et al. 2009, ApJ, 696, 713

Strolger, L., Dahlen, T., \& Riess, A. G. 2010, ApJ, 713, 32

Strolger, L., Riess, A. G., Dahlen, T., et al. 2004, ApJ, 613, 200

Sullivan, M., Howell, D. A., Perrett, K., et al. 2006, AJ, 131, 960

Tonry, J. L., Schmidt, B. P., Barris, B., et al. 2003, ApJ, 594, 1

Toonen, S., Nelemans, G., \& Portegies Zwart, S. 2012, A\&A, 546, A70

Totani, T., Morokuma, T., Oda, T., Doi, M., \& Yasuda, N. 2008, PASJ, 60,1327

Umetsu, K., Medezinski, E., Nonino, M., et al. 2012, ApJ, 755, 56

Vernet, J., Dekker, H., D’Odorico, S., et al. 2011, A\&A, 536, A105

Wang, B., \& Han, Z. 2012, NewAR, 56, 122

Wang, B., Li, X.-D., \& Han, Z.-W. 2010, MNRAS, 401, 2729

Wang, L., Strovink, M., Conley, A., et al. 2006, ApJ, 641, 50

Webbink, R. F. 1984, ApJ, 277, 355

Whelan, J., \& Iben, I., Jr. 1973, ApJ, 186, 1007

Wuyts, S., Labbé, I., Schreiber, N. M. F., et al. 2008, ApJ, 682, 985

York, D. G., Adelman, J., Anderson, J. E., Jr., et al. 2000, AJ, 120, 1579

Yüksel, H., Kistler, M. D., Beacom, J. F., \& Hopkins, A. M. 2008, ApJL, 683, L5 (Y08)

Yungelson, L. R. 2010, AstL, 36, 780 